Revue des patrimoines

4 | 2004

Les réseaux de la villégiature

\title{
La place de l'automobile dans le développement des stations
}

Paul Smith

\section{(2) OpenEdition}

Journals

Édition électronique

URL : http://journals.openedition.org/insitu/1999

DOI : 10.4000/insitu.1999

ISSN : 1630-7305

Éditeur

Ministère de la Culture

Référence électronique

Paul Smith, «La place de l'automobile dans le développement des stations », In Situ [En ligne], 4 | 2004, mis en ligne le 01 mars 2004, consulté le 07 septembre 2020. URL : http://journals.openedition.org/ insitu/1999; DOI : https://doi.org/10.4000/insitu.1999

Ce document a été généré automatiquement le 7 septembre 2020

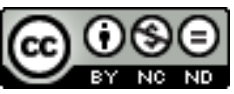

In Situ Revues des patrimoines est mis à disposition selon les termes de la licence Creative Commons Attribution - Pas d'Utilisation Commerciale - Pas de Modification 4.0 International. 


\title{
La place de l'automobile dans le développement des stations
}

\author{
Paul Smith
}

\section{Les réseaux de la villégiature : l'automobile}

1 Pour qui s'intéresse à l'histoire et au patrimoine des lieux de villégiature, la compréhension des réseaux de transport qui relient ces lieux à leurs clientèles est un point de départ évident et structurant, comme pour le patrimoine industriel, où il importe toujours de savoir comment les matières premières parviennent à l'usine. En considérant cette question - comment les curistes sont amenés à l'eau, comment les baigneurs arrivent aux flots -, si l'on n'oublie pas l'importance primitive de la navigation à vapeur ${ }^{1}$, c'est surtout l'apport des chemins de fer qui est bien documenté et étudié : l'histoire des compagnies, la création et la ramification des lignes sur lesquelles les stations s'embranchent, la construction de la première gare, l'inauguration de la deuxième gare, les affiches publicitaires ${ }^{2}$, la part des compagnies dans l'aménagement de la station, voire dans ses opérations de lotissement... 
Figure 1

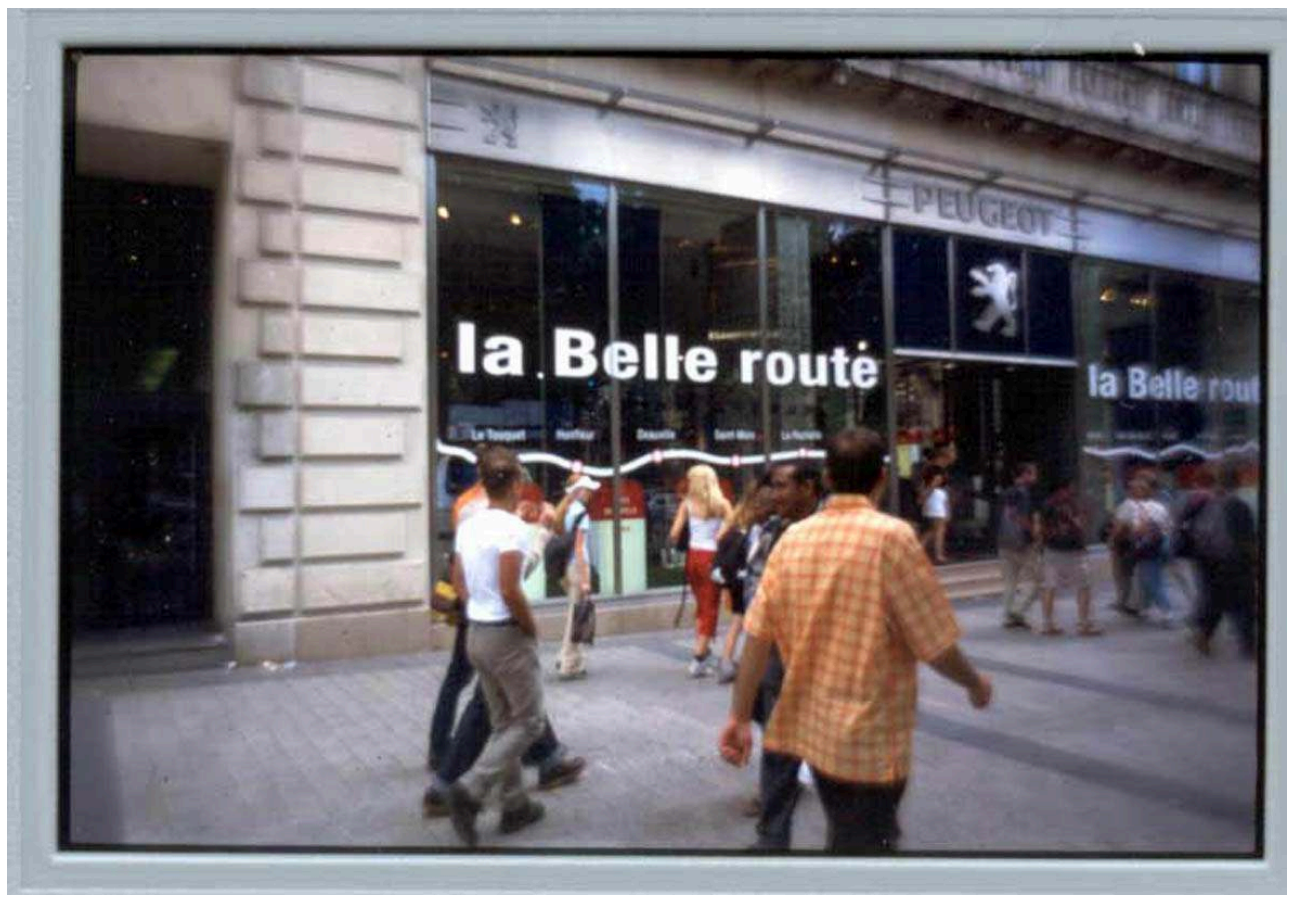

«La Belle Route », magasin Peugeot sur les Champs-Elysées.

Phot. P. Smith, 2002 ( ) P. Smith.

2 Mais si le chemin de fer est fondateur du tourisme au XIX siècle, c'est l'automobile qui conduit son développement au XX' ${ }^{\mathrm{e}}$. L'attraction mutuelle et maintenant séculaire entre l'automobile et la station balnéaire s'est illustrée récemment à la vitrine du magasin Peugeot sur les Champs-élysées. Vue par les services de communication du grand constructeur, cette attraction était évoquée par « la Belle Route ", une route nationale reliant d'un trait Le Touquet à Hossegor, Deauville à Biarritz, en passant par La Baule (fig. $\left.\mathbf{n}^{\circ} \mathbf{1}\right)$. Cette route imaginaire des débuts de l'été 2002 présente l'avantage ici de nous faire remonter aux Champs-élysées, première vitrine, à la Belle époque, d'une industrie en première vitesse ${ }^{3}$. L'industrie de l'automobile est née à Paris. Le Grand Palais, avec ses salons annuels à partir de $1901^{4}$, puis les premiers magasins permanents sur les Champs et sur l'avenue de la Grande-Armée, mettaient ses produits en scène et lui servaient de points de vente. Les usines de construction se rassemblent alors en banlieue ouest sur les rives de la Seine, où les terrains sont moins chers. Dans les 36 communes qui forment, depuis 1964, le département des Hauts-de-Seine, on a pu inventorier pas moins de trois cents entreprises de construction automobile, sans compter les carrossiers, équipementiers et accessoiristes ${ }^{5}$. On pense bien sûr à Louis Renault à Billancourt, mais on peut se souvenir aussi, et avant lui, de De Dion-Bouton à Puteaux, d'Alexandre Darracq à Suresnes, de Fernand Charron à Courbevoie ou d'Adolphe Clément, dit Clément-Bayard, à Levallois-Perret, pour ne citer que quelques grands.

3 Cette concentration de l'industrie naissante en région parisienne relève en partie de la disponibilité d'une main-d'œuvre qualifiée, rompue dans la capitale aux métiers de la carrosserie hippomobile, de la construction mécanique ou de la fabrication de bicyclettes. Mais elle s'explique surtout par la proximité d'une clientèle domiciliée majoritairement dans les arrondissements à l'ouest de Paris, une clientèle aux allures 
emmitouflées de «sportsmen », d'amateurs de " la vie au grand air », forcément aisés. Car la voiture automobile coûte très cher : le premier prix dans le catalogue Panhard et Levassor de 1892 est 5000 francs, soit l'équivalent de quatre ou cinq années de salaire d'un ouvrier métallurgiste de la région parisienne. Et ce premier prix n'est qu'un début. Il faut d'abord faire carrosser le véhicule dont on n'a payé que le châssis avec le moteur. Ensuite, à moins d'être centralien ou garagiste, il faut encore pouvoir gager un chauffeur et le loger.

Figure 2

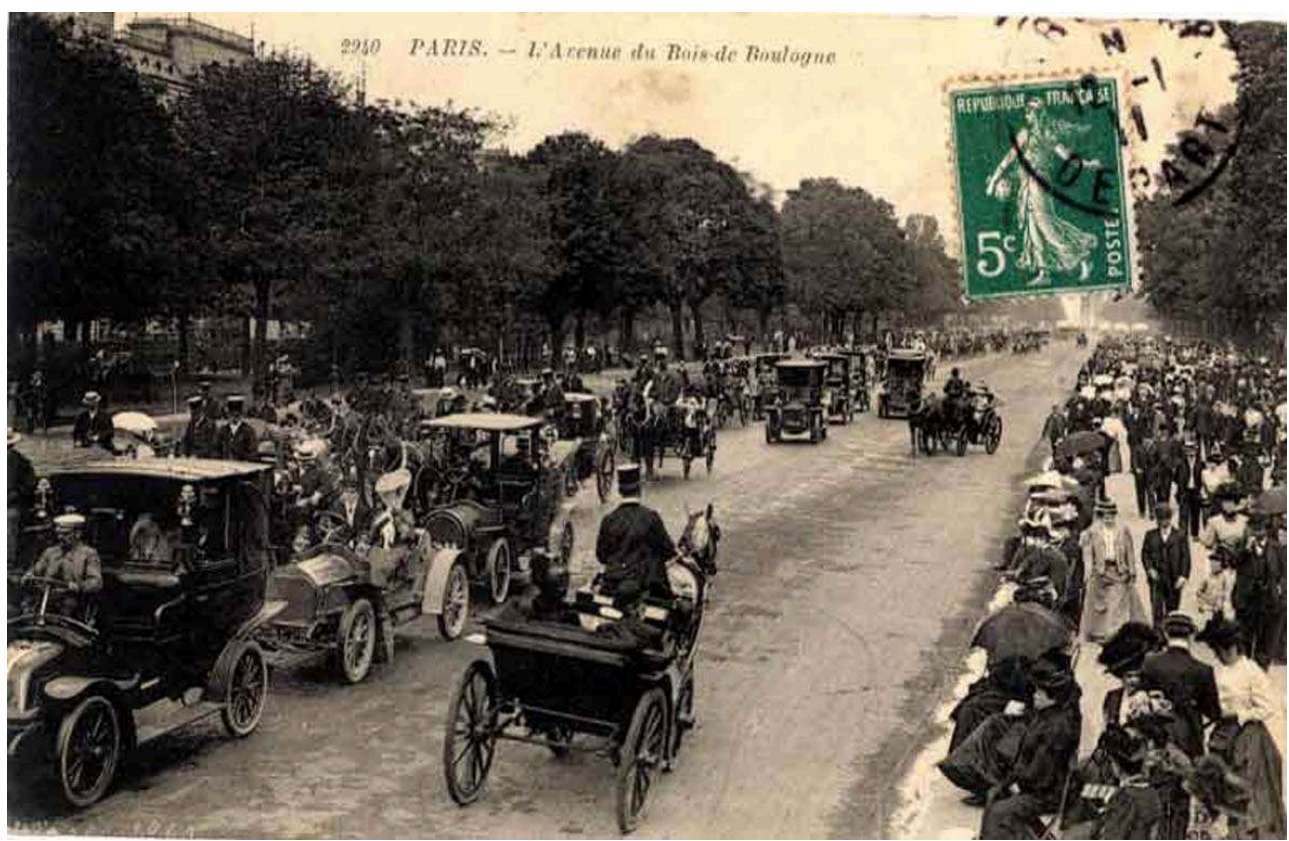

Paris, l'avenue du Bois-de-Boulogne, carte postale envoyée en 1911. Coll. part.

4 Une fois le fabuleux jouet acquis, qu'en faire? Où le faire voir? Où se retrouver? Où aller? Dans un premier temps, vers le bois de Boulogne, destination traditionnelle des équipages mondains (fig. $\mathbf{n}^{\circ}$ 2), mais assez rapidement, toujours à l'ouest, vers la côte normande, à Trouville par exemple, cette « Reine des Plages » lancée sous la Monarchie de Juillet et rendue accessible en train, par la Compagnie de l'Ouest, en $1863^{6}$. L'impact précoce du phénomène automobile sur les stations de la côte normande est reconnu de longue date, déjà remarqué par Gabriel Désert dans son ouvrage de 1983 traitant des plages normandes du Second Empire aux Années Folles ${ }^{7}$. Deauville, la station voisine et rivale de Trouville fondée sous le Second Empire par le duc de Morny et son médecin, puis relancée à la veille de la Première Guerre mondiale par Eugène Cornuché, deviendra l'incontestable capitale balnéaire de l'automobile. Les remarques qui suivent sur la place de l'automobile dans les réseaux de la villégiature et sur ses influences sur l'architecture des stations s'inspireront surtout de cet exemple deauvillais, que j'ai eu par ailleurs l'occasion d'étudier?.

\section{Deauville}


Figure 3

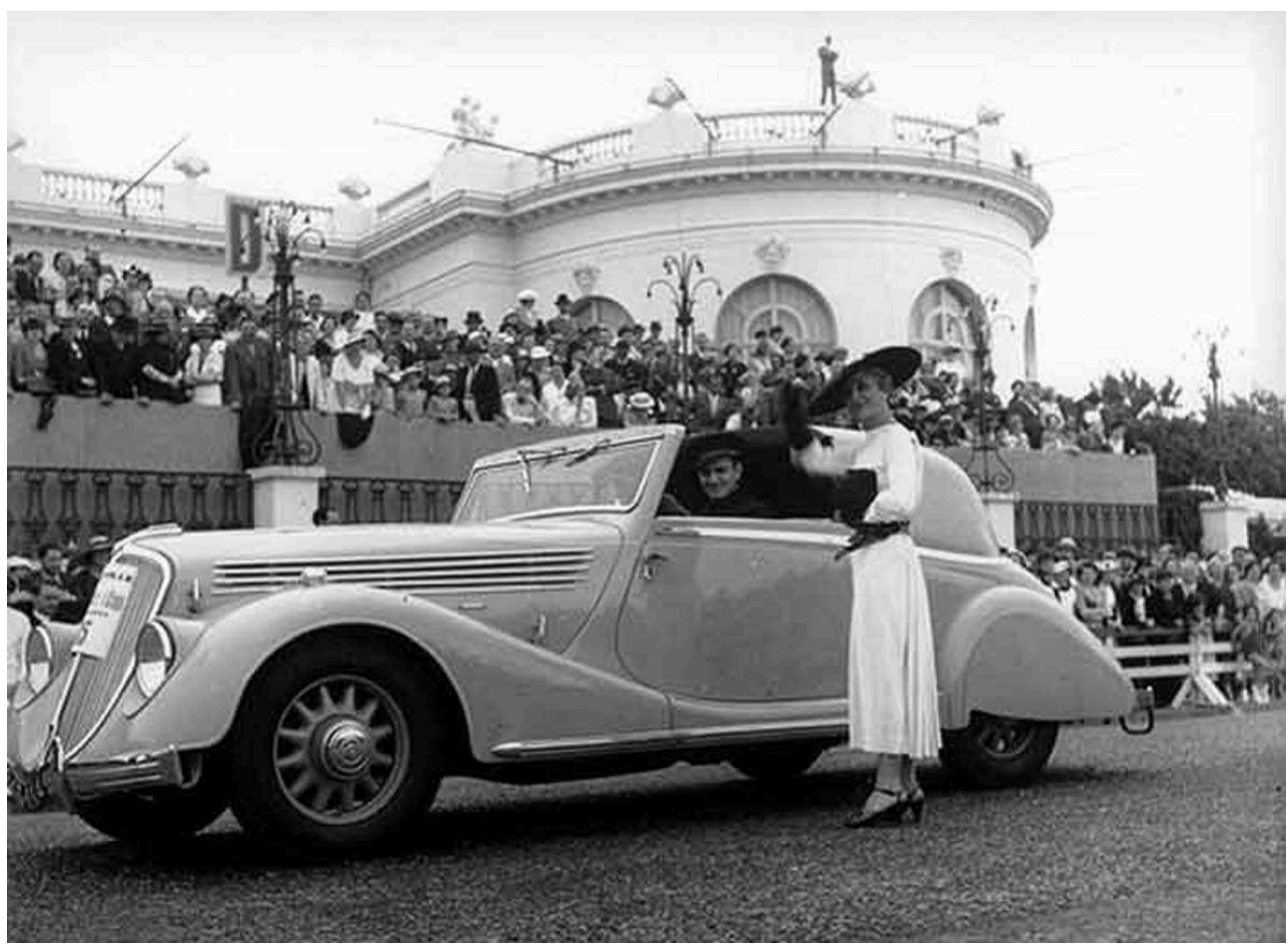

Deauville le 13 juillet 1936, concours d'élégance « Viva grand sport » devant la terrasse du casino.

Photothèque Renault ( ) Renault communication. Droits réservés.

5 En effet, la «résurrection» de Deauville-la-morte aux cours des trois années qui précèdent la Première Guerre mondiale et l'essor de sa réputation dans les années 1920 comme station par excellence de l'argent élégant et cosmopolite sont en prise directe avec le phénomène automobile: ses produits, ses industriels, ses bénéfices et les différentes formes de son autocélébration. A posteriori, et en comparaison surtout avec les ruelles escarpées de Trouville, l'urbanisme concerté de Deauville, sa voirie large, droite et plane, tirée au cordeau par les premiers promoteurs des années 1860, semble mieux adapté aux besoins modernes de l'automobilisme. Même le retrait imprévu de la laisse de haute mer pendant l'hiver 1874-1875 contribuera à faire de la terrasse deauvillaise une piste propice aux courses, aux défilés et aux concours d'élégance (fig. $\mathbf{n}^{\circ}$ 3), léguant par la même occasion de généreux espaces aménageables en équipements sportifs et en allées pour le rangement de voitures toujours plus nombreuses. 
Figure 4

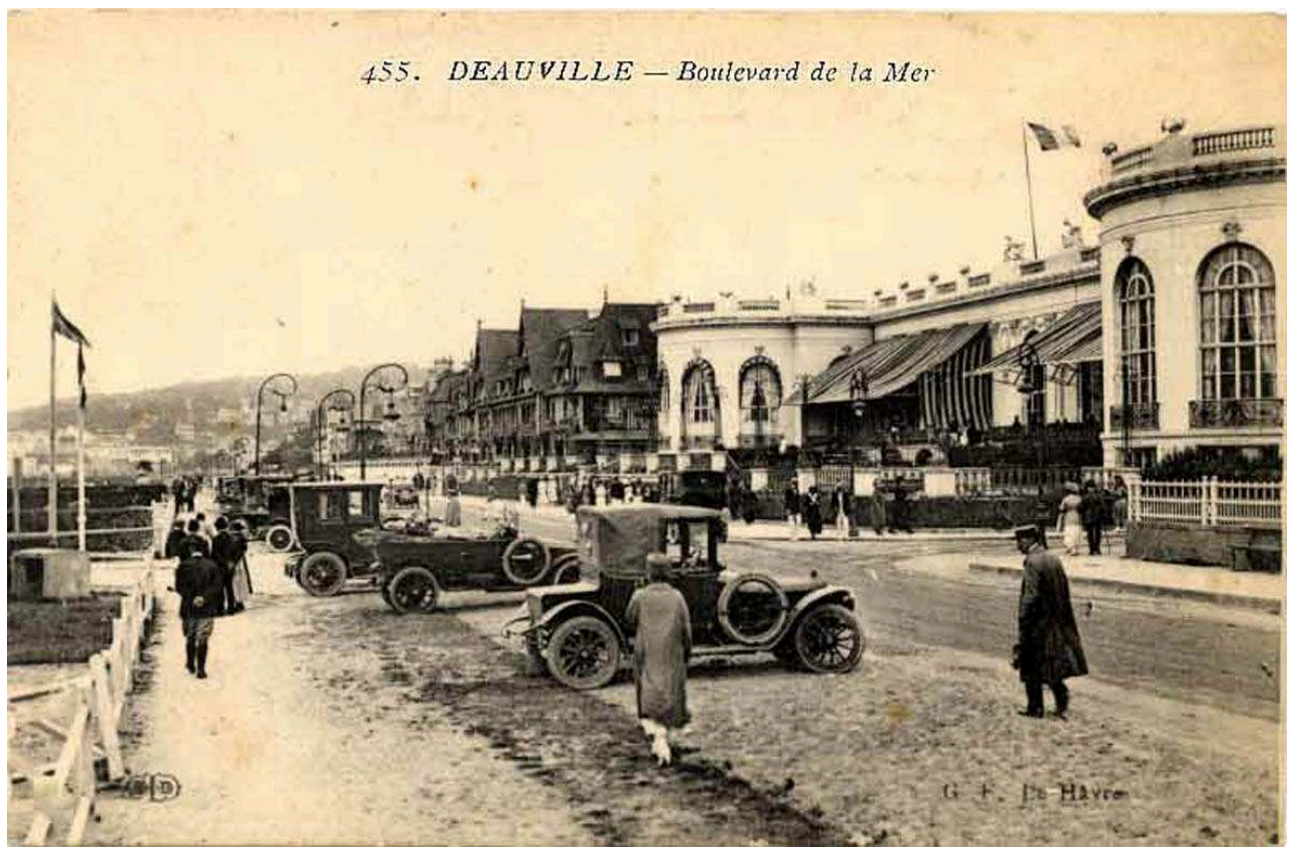

Deauville, boulevard de la Mer, l'actuel boulevard Eugène-Cornuché, devant le casino et l'hôtel Normandy, carte postale début du XXe siècle. Coll. part.

6 En milieu urbain, celles-ci s'accompagnent déjà de leurs effets pervers familiers : excès de vitesse, accidents, bruit et bouchons (fig. $n^{\circ}$ 4).

Figure 5

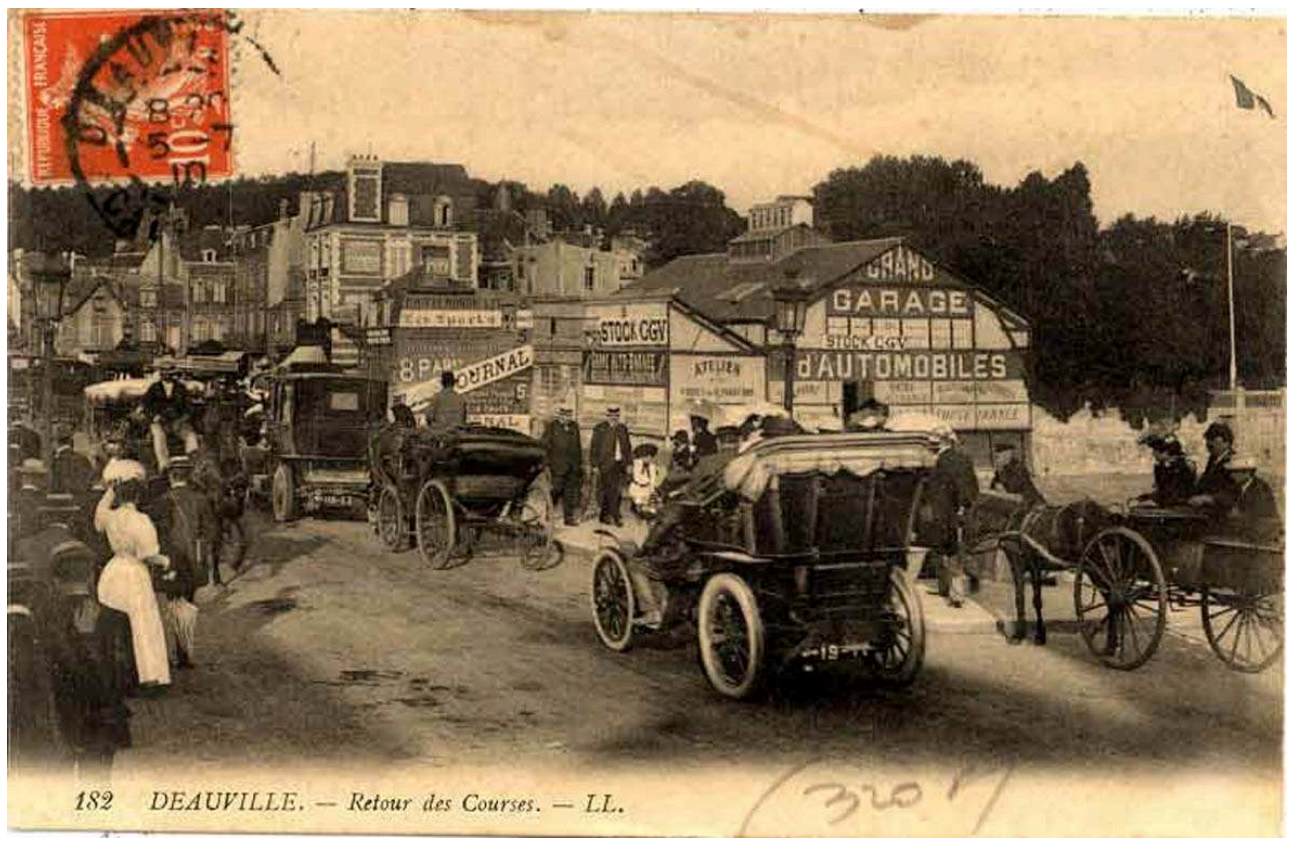

Deauville, Retour des courses, carte postale envoyée en juillet 1910. Le garage « Stock C.G.V. » est celui des constructeurs Fernand Charron, Léonce Girardot et Emile Voigt, entreprise fondée en 1901. Coll. part.

7 En juillet 1899, le maire de Trouville interdit la circulation automobile dans la rue des Bains et sur la route de la Corniche ${ }^{10}$, et en août 1909, celui de Deauville, Désiré Le Hoc, 
est amené à signer l'arrêté municipal suivant : "Article 1 : Les voitures automobiles ne devront pas dépasser la vitesse de 15 kilomètres à l'heure dans l'intérieur de la ville ; Article 2 : Est interdit l'emploi des sirènes et des sifflets comme appareils avertisseurs ${ }^{11}$ ». L'avenue de Villers à Deauville (l'actuelle avenue de la République) sera l'une des toutes premières au monde à connaître cette manifestation essentielle de l'automobile, l'embouteillage (fig. $\mathbf{n}^{\circ}$ ), attesté lors du retour des courses par des taux de participation chiffrés avec précision : 2218 automobiles, contre seulement 896 voitures hippomobiles, pour les journées des courses hippiques de 1909; 3613 en $1912^{12}$.

8 Les premières voitures à être vues sur la côte sont sans doute celles de courses automobiles, comme par exemple cette course de vitesse Paris-Trouville du 14 août 1897, départ Saint-Germain, gagnée en 3 heures 51 minutes et 56 secondes par Jamin sur une voiturette Bollée. Cette compétition fait partie d'une série de courses de ville à ville organisées entre 1894 et 1903, épreuves souvent meurtrières par lesquelles l'industrie automobile d'avant les réseaux de vente constitués faisait connaître les promesses de sa marchandise et ses noms de marque. Le lundi 16 août, la course est suivie sur la plage de Deauville, et sous la pluie, d'un défilé fleuri de tous les véhicules ayant pris part à la manifestation, défilé ouvert « à toutes les voitures d'amateurs, sans distinction ${ }^{13}$ ». Les 25 et 26 août 1899, c'est le "Grand Handicap Omnium », une course originale aux départs soigneusement échelonnés, mettant en concurrence coureurs à pied, chevaux, bicyclettes, voiturettes, motocycles et automobiles. Elle est remportée par une voiture attelée, la première automobile, une Mors pilotée par A. Debray, n'arrivant à Trouville qu'en quatrième position après 4 heures et 58 minutes de route. Début septembre 1901, ce sont les courses du mille et du kilomètre lancé qui ont lieu sur la terrasse de Deauville. Le 9 septembre 1903 s'y dispute une course de 500 mètres, départ et arrivée arrêtés, les roues avant et arrière devant s'immobiliser de part et d'autre de la ligne du but.

9 Autour de l'authentique contenu sportif de ces événements et au-delà du montant coquet des prix $^{14}$, on peut entrevoir d'autres enjeux. Pour les coureurs et les constructeurs, qui ne disposent pas encore de circuits fermés ni de creusets de vitesse, il s'agit bien de bancs d'essais physiques et mécaniques. Les 500 mètres de 1903, par exemple, mettent à l'épreuve les qualités de freinage des véhicules, « actuellement l'un des gros problèmes de l'automobilisme ${ }^{15}$ ". Pour ces mêmes industriels et pour d'autres promoteurs de l'automobile, rassemblés à partir de 1896 dans le très aristocratique et très parieur Automobile Club de France, installé depuis 1900 place de la Concorde, il s'agit d'encourager plus généralement l'industrie naissante. C'est l'intérêt particulier que trouve au défilé fleuri du 16 août 1897 le quotidien Le Journal, sponsor de la course de l'avant-veille avec l'Automobile Club de France : " c'est en effet ce qui frappe le plus l'imagination des foules et ce qui peut contribuer le plus efficacement au triomphe de l'automobilisme. Il faut montrer au public, en évoluant sous ses yeux, que nos véhicules ne présentent aucun danger, qu'ils se manient et se dirigent facilement ${ }^{16}$ ». Sans oublier l'intérêt évident des organes de la presse parisienne - des chroniques assurées et haletantes pendant la morte-saison parlementaire -, imaginons les avantages pour l'économie de la station d'accueil : 6 000, 10 000, 20000 spectateurs, des marchands de fleurs mis au pillage, des hôtels regorgeant de monde et tout ceci, qui plus est, en dehors de la semaine des courses hippiques. L'augmentation de la durée de la saison et la diversification de ses attractions, voilà, en effet, l'une des ambitions constantes des 
équipes municipales des stations balnéaires, clairement énoncée par exemple dans les statuts du syndicat d'initiative et de propagande de Deauville, créé fin $1911^{17}$.

Figure 6

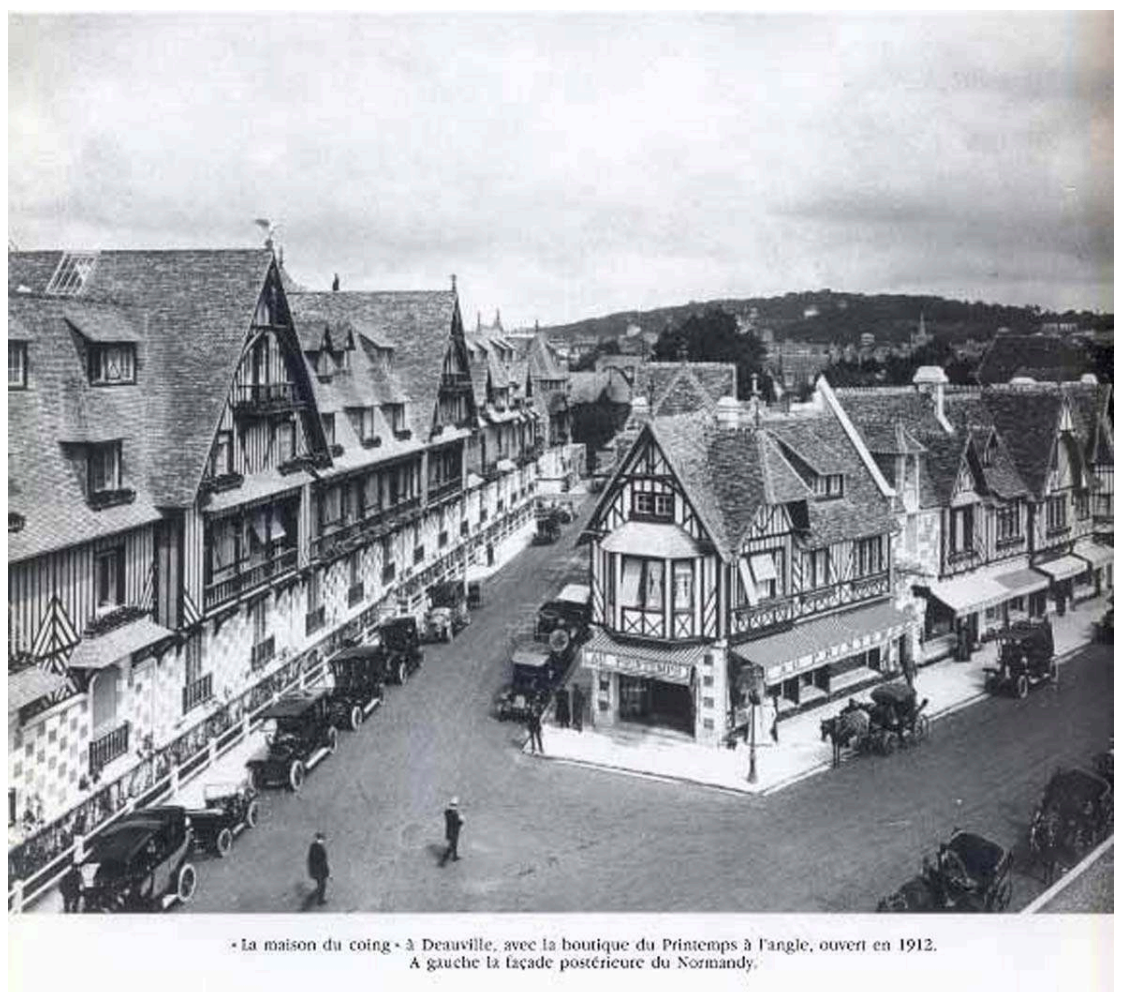

Deauville en 1913, vue prise depuis les toits du casino. La "maison du coing », construite par Georges Wybo, en collaboration avec E. Mauclair, pour les Grands Magasins du Printemps, la première succursale du Printemps en province, ouverte seulement durant l'été. A gauche, la façade postérieure du Normandy, construit par l'architecte parisien Théo Petit de 1911 à 1912.

Photo Georges Wybo, I.F.A., Fonds Georges Wybo (177 IFA).

Quoi de moins étonnant que tous ces intérêts viennent à se conjuguer dans les rédactions parisiennes ou à la Potinière de Deauville, même si cette mouvante sociabilité balnéaire, ces réseaux entremêlés d'hommes, de femmes et de fortunes, de cercles, de connaissances, de carnets d'adresses et de commandes, échapperont toujours en grande partie à notre curiosité patrimoniale. Mais prenons par exemple Eugène Letellier, entrepreneur en travaux publics d'origine belge, commanditaire du Journal, dont le siège, au 100 rue de Richelieu, est surélevé par l'architecte Georges Wybo en $1910^{18}$. Letellier est propriétaire à Hennequeville et maire de Trouville de 1904 à 1910. Son fils Henri, directeur du Journal, sera maire de Deauville entre 1925 et $1928^{19}$. Les Letellier sont parmi les principaux bailleurs de fonds de la nouvelle Société des Hôtels et Casino de Deauville, fondée en décembre 1910 par Eugène Cornuché, et dont Georges Wybo sera le principal architecte. En avril 1909, Cornuché, alors patron de Maxim's, cantine de l'Automobile Club de France, aurait été introduit comme administrateur délégué à la Société des Casinos de Trouville par Alexandre Darracq, intéressé à la Société trouvillaise en règlement d'une créance impayée ${ }^{20}$. Et avec Darracq, l'un des quatre plus gros constructeurs automobile de l'époque, et mélangées à des fortunes plus anciennement acquises - Menier dans le chocolat, Lebaudy dans le sucre, Rothschild dans la haute finance...- bien d'autres figures liées aux nouveaux 
modes de locomotion se feront remarquer à Trouville et à Deauville à la Belle époque : Robert Delaunay-Belleville, Fernand Charron, les frères Louis et Emile Mors, André Citroën (alors directeur des usines de ces deux derniers), Decauville, Armand Peugeot et l'aviateur Alberto Santos-Dumont ${ }^{21}$, sans oublier E. Deutsch de la Meurthe, membre de la famille pétrolière et du comité d'honneur du syndicat d'initiative de Deauville. Pour l'auteur d'un guide de 1913, ce sont eux les découvreurs du nouveau Deauville: «Les Deutsch, les Peugeot, les Mors s'aperçurent que Deauville existait réellement et s'y fixèrent pour leur villégiature d'été22 ", et avec eux... leurs véhicules (fig. $\mathbf{n}^{\circ}$ 6).

Figure 7

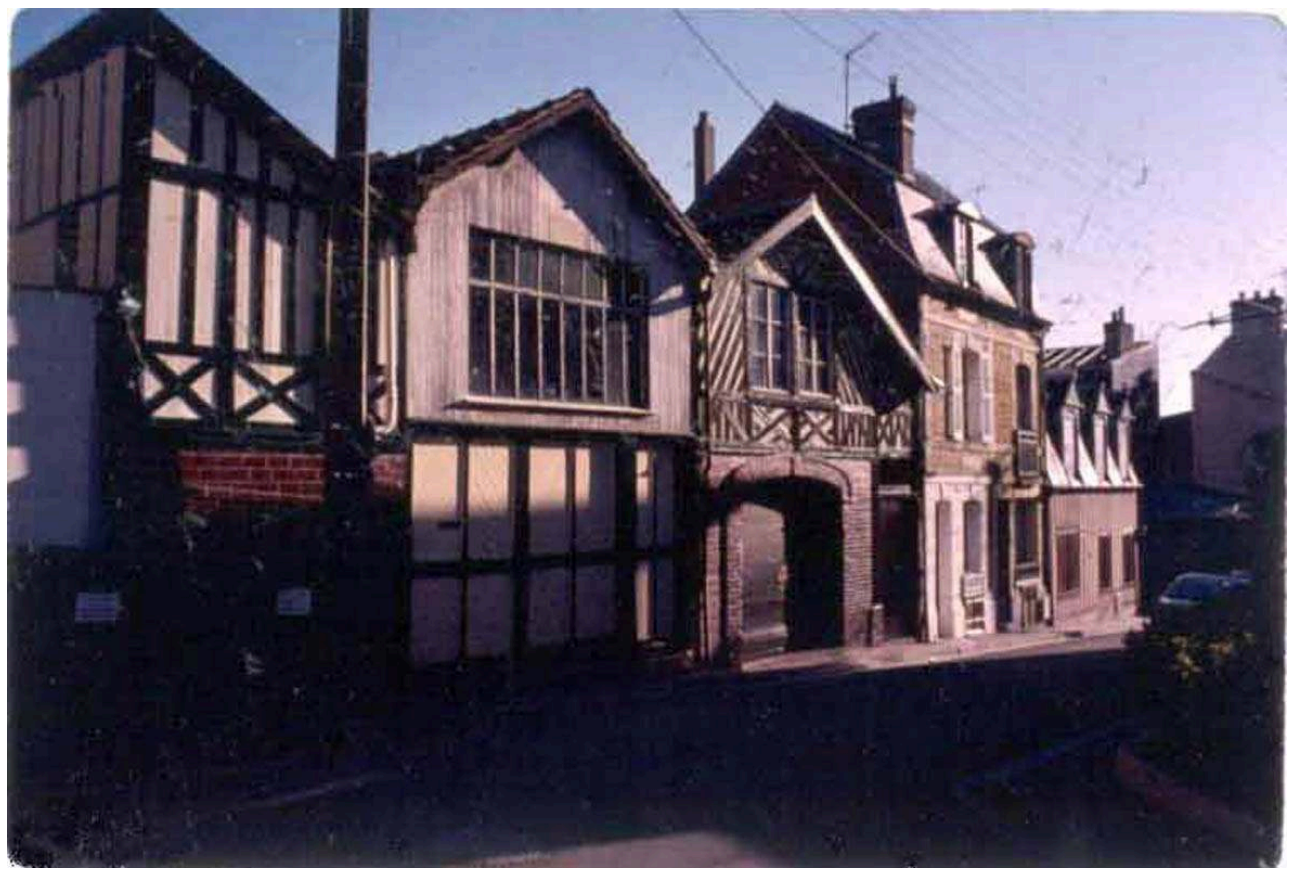

Remises et garages, rue du Général-Leclerc à Trouville. Phot. P. Smith, 1997 @ P P. Smith.

$11 \mathrm{Si}$, aujourd'hui, on ne craint pas trop de laisser sa voiture dehors pendant la nuit, ce n'était pas le cas à la Belle époque. L'un des premiers impacts du nouveau moyen de locomotion en termes architecturaux fut donc l'apparition du garage particulier. Dans bien des cas, dans les communs des grandes villas, d'anciennes remises et écuries pouvaient offrir sans mal l'abri nécessaire aux nouvelles voitures sans cheval. A Trouville, le long de l'ancienne rue d'Orléans (rue du Général-Leclerc) s'aligne encore une belle sélection de remises hippomobiles devenues garages automobiles (fig. $\mathbf{n}^{\circ} \mathbf{7}$ ). 
Figure 8

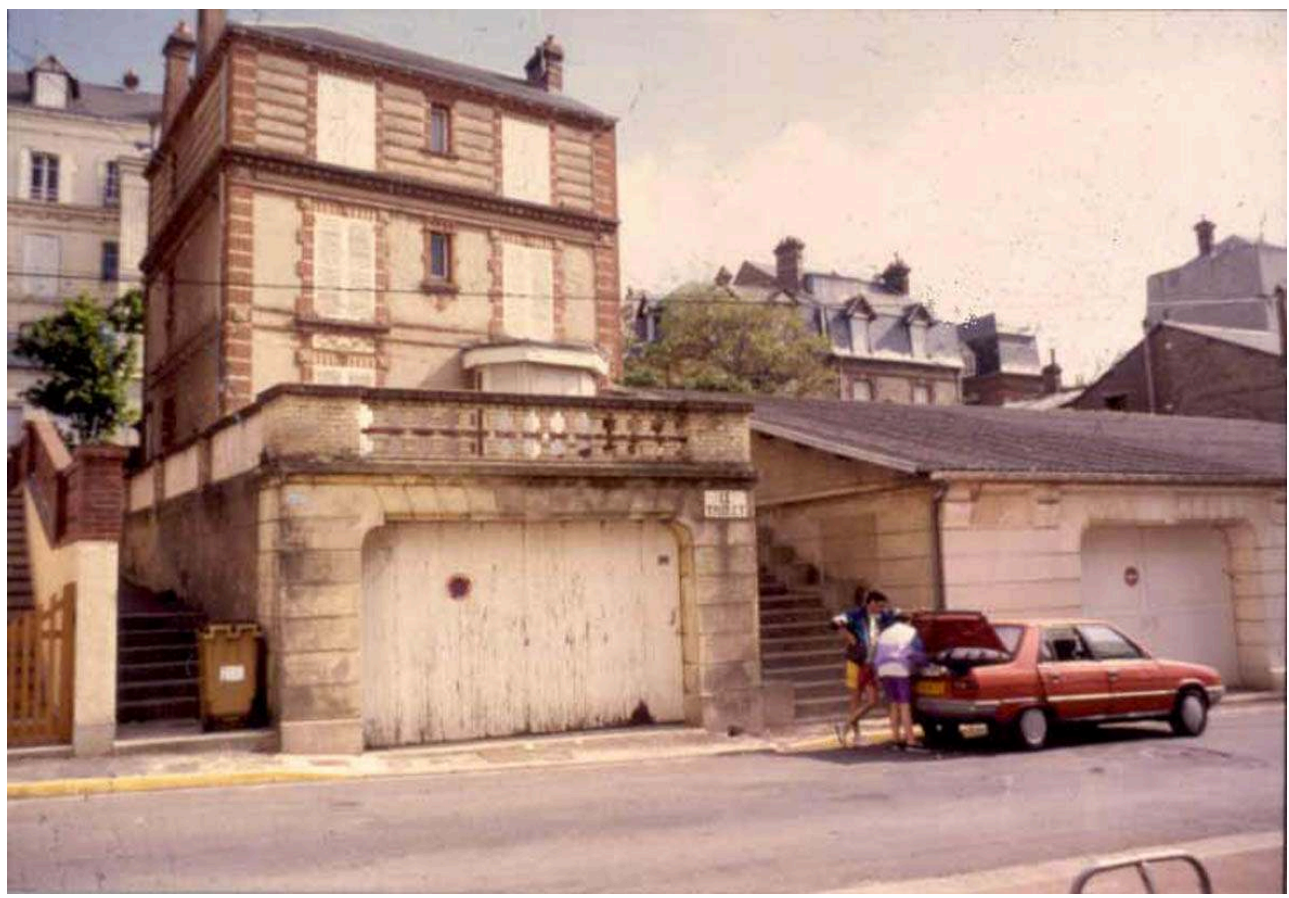

Houlgate, garages particuliers dans la rue des Bains.

Phot. P. Smith, 1995 ( ) P. Smith.

12 Progressivement, toutefois, le garage particulier doit trouver sa place comme partie intégrante du programme de la villa balnéaire (fig. $\mathbf{n}^{\circ} \mathbf{8}$ ). 
Figure 9

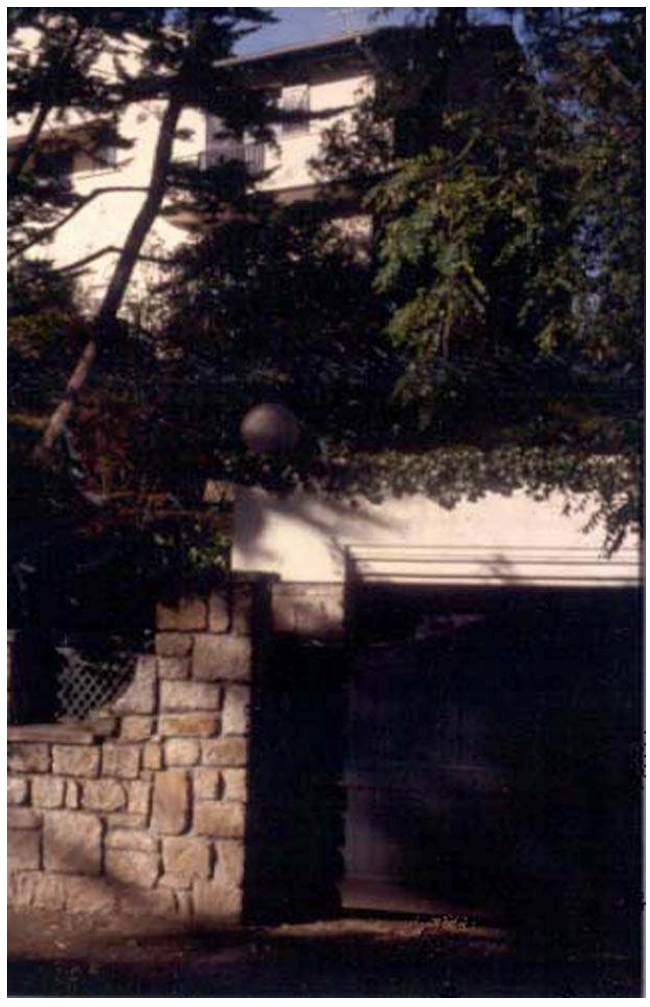

La Baule, garage de la villa Aktina.

Phot. P. Smith, 2002 (c) P. Smith.

13 Les hôtels se doivent également d'offrir à leur clientèle des locaux pour le remisage des autos. Dès 1901, le Grand Hôtel Bellevue et le Grand Hôtel de la Terrasse à Trouville disposent, d'après les guides pratiques Conty, de "garages pour automobiles " (fig. $n^{\circ}$ 9). 
Figure 10

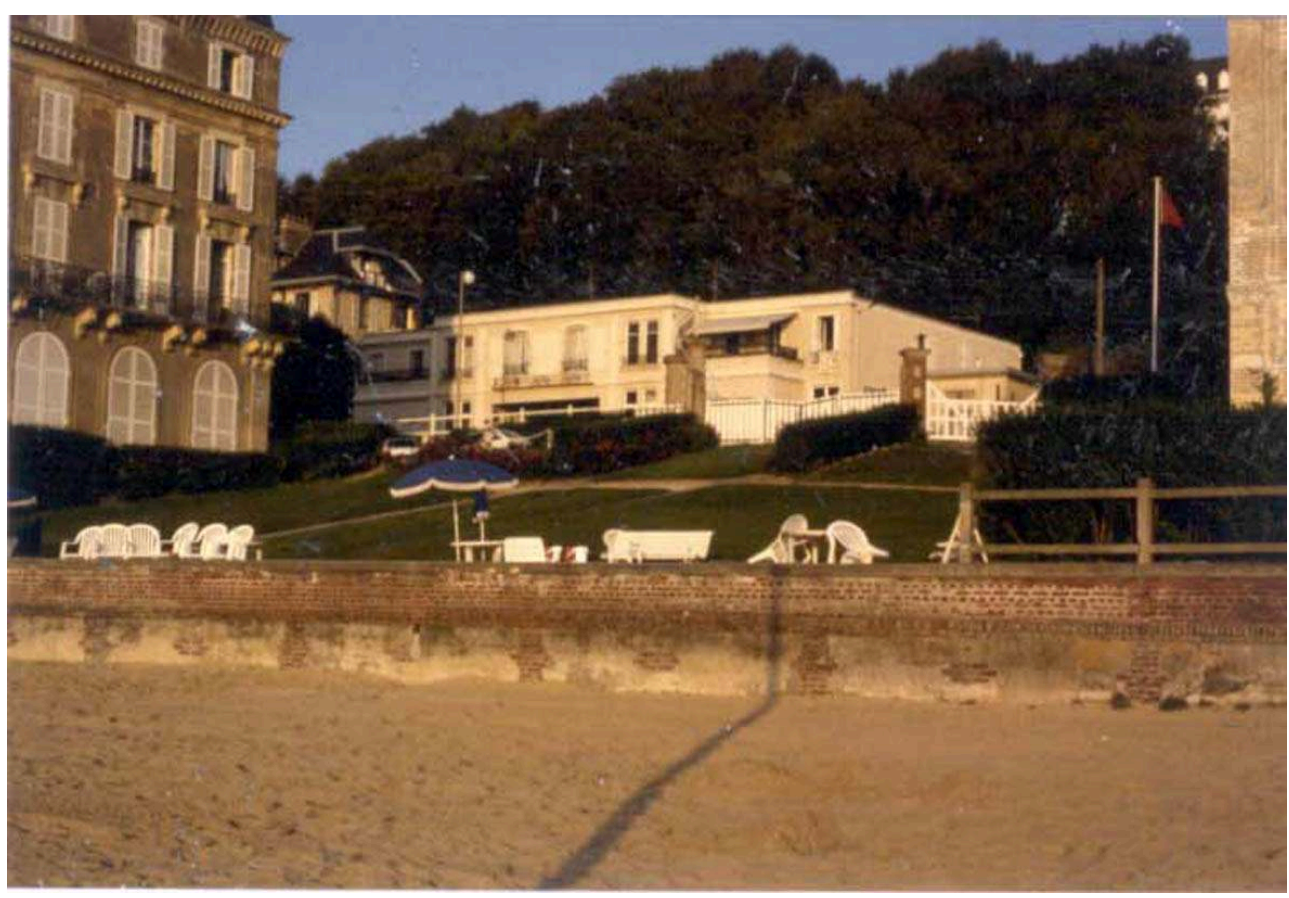

Garage collectif de l'hôtel des Roches Noires (à gauche de l'image).

Phot. P. Smith, 1992 (c) P. Smith.

En 1904, l'Hôtel de Paris à Trouville propose «remises et écuries, et garages d'automobiles ${ }^{23}$.

15 Aménagé par Rob Mallet-Stevens en 1924, le célèbre Hôtel des Roches Noires (bien connu des amis de Marguerite Duras et de Dominique Delaunay) se dote dans les années 1920 d'un grand garage collectif, avec logements de chauffeurs à l'étage (fig. $\mathbf{n}^{\circ} \mathbf{1 0}$ ).

16 Autre nouveauté : l'apparition du garage dans son sens d'atelier d'entretien et de réparation, avec mécaniciens, fosses et stocks de pièces détachées et de pneus. Les automobiles sont encore fragiles et loin des usines de la banlieue où l'on faisait généralement effectuer les réparations. Les premiers garages à Deauville - on en dénombre sept en 1907 - sont rassemblés surtout à proximité de la gare et notamment sur la presqu'île qui sépare le bassin à flot de la Touques, zone industrielle avec ses entrepôts de charbon, de bois et de glace. 
Figure 11

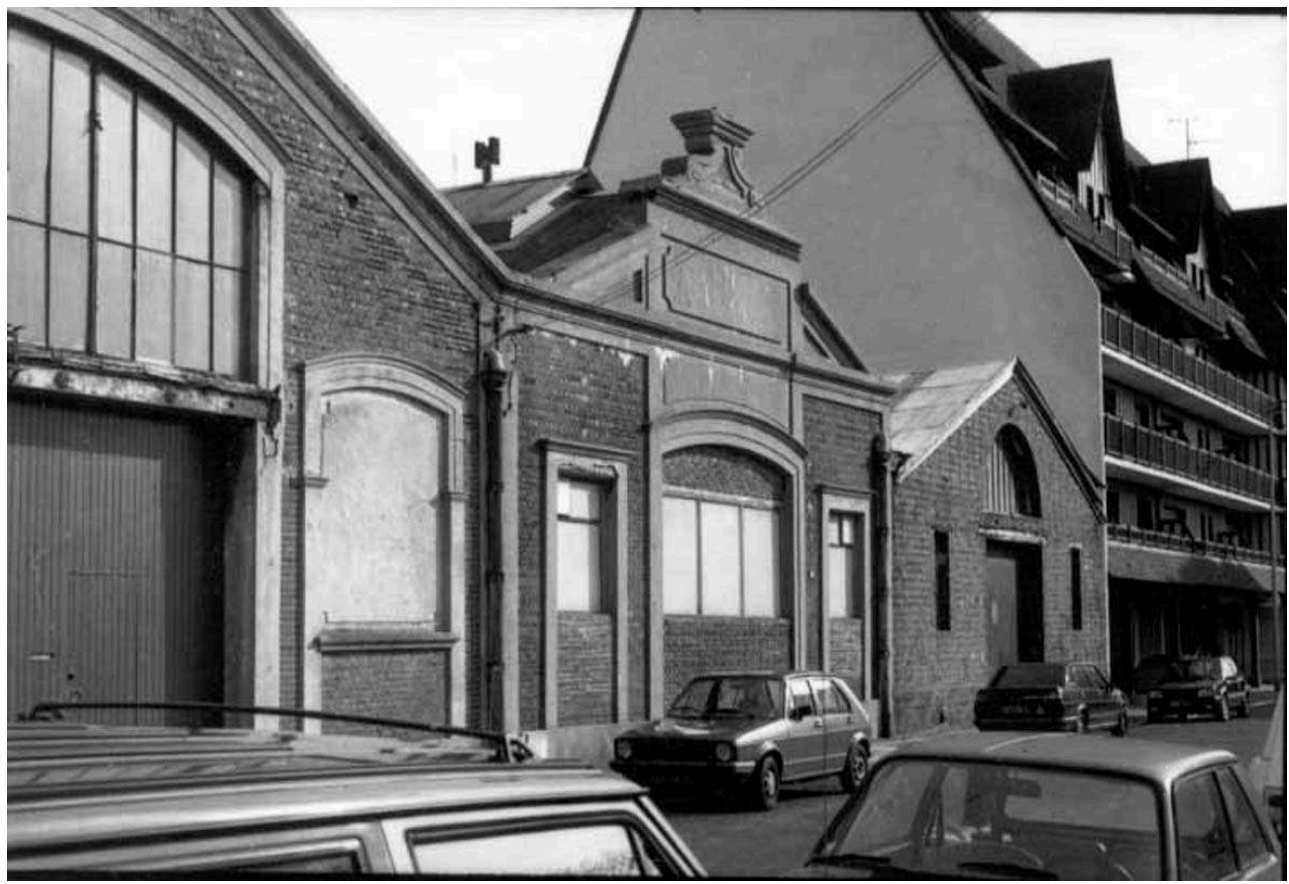

Ancien garage rue Thiers à Deauville, sur la presqu'île en face de la gare (actuellement détruit)

Phot. P. Smith, 1991 (c) P. Smith

17 Garage de la Rotonde, Auto-Palace, Grand Garage Central H. Chéringou, puis Dumontier, puis Dauquaire et Bruneau, Garage de l'Arrivée Fargette et Couyère... les raisons sociales de ces entreprises indépendantes ont parfois la vie brève, mais, du moins au début des années 1990, quelques frontons ou pignons d'ateliers remontant à la Belle époque ou aux années 1920 et donnant sur la rue Thiers témoignaient encore, avec quelques photographies anciennes, de la première occupation automobile de ce territoire (fig. $\mathbf{n}^{\circ} \mathbf{1 1}$ ). Hormis les réparations, l'entretien et la vente d'huile et d'essence - cette dernière étant également assurée par l' épicerie parisienne de Félix Lavot, rue du Casino -, ces établissements pouvaient offrir aussi des boxes pour le remisage de voitures particulières, des chambres pour chauffeurs, ainsi que des services de location. L'automobile commence à sortir ainsi de ses circuits sportifs pour devenir un moyen de promenade, attraction parmi d'autres dans la surabondance de plaisirs que subventionnent et programment ensemble syndicats d'initiative, édiles et casinos. Pour la saison 1913, le syndicat d'initiative de Deauville fait éditer un guide particulier, Cent kilomètres autour de Deauville, une dizaine d'itinéraires à travers la Normandie plantureuse, dus à la plume de Mademoiselle S. Turgis : "Les jours de courses, par exemple, tout va bien, pourvu que le terrain soit bon et que le temps permette l'exhibition de jolies toilettes, mais le lendemain? Le polo, le tennis, le flirt... ne suffisent pas, on se lasse de tout. Reste l'auto... Où aller ? ?"

18 Les premiers emplois sportifs, touristiques ou oisifs de l'auto ne doivent pas masquer non plus l'émergence autour de 1905 de son usage comme moyen de transport essentiellement utilitaire: "la plupart des voitures de notre département appartiennent à des hommes qui s'en servent pour l'exercice de leur industrie et sont donc un moyen de travail» précise le premier numéro de la revue mensuelle de l'Automobile Club de Basse-Normandie, fondée à Caen en juillet 1912. L'emploi de 
l'automobile en versions spécifiquement utilitaires va, lui aussi, croissant : camions de livraison pour la succursale deauvillaise du magasin du Printemps, arroseuse automobile De Dion Bouton acquise par la ville en 1911 pour le service de la voirie ${ }^{25}$, voitures de place à la gare... En 1912, un service d'autobus fait l'aller-retour entre le casino et la gare ; d'autres véhicules - «les confortables autobus de la Compagnie des Messageries Automobiles » - relient Deauville à Honfleur, « la plus belle excursion de la région : on accepte les bagages et les bicyclettes ${ }^{26} »$.

Figure 12

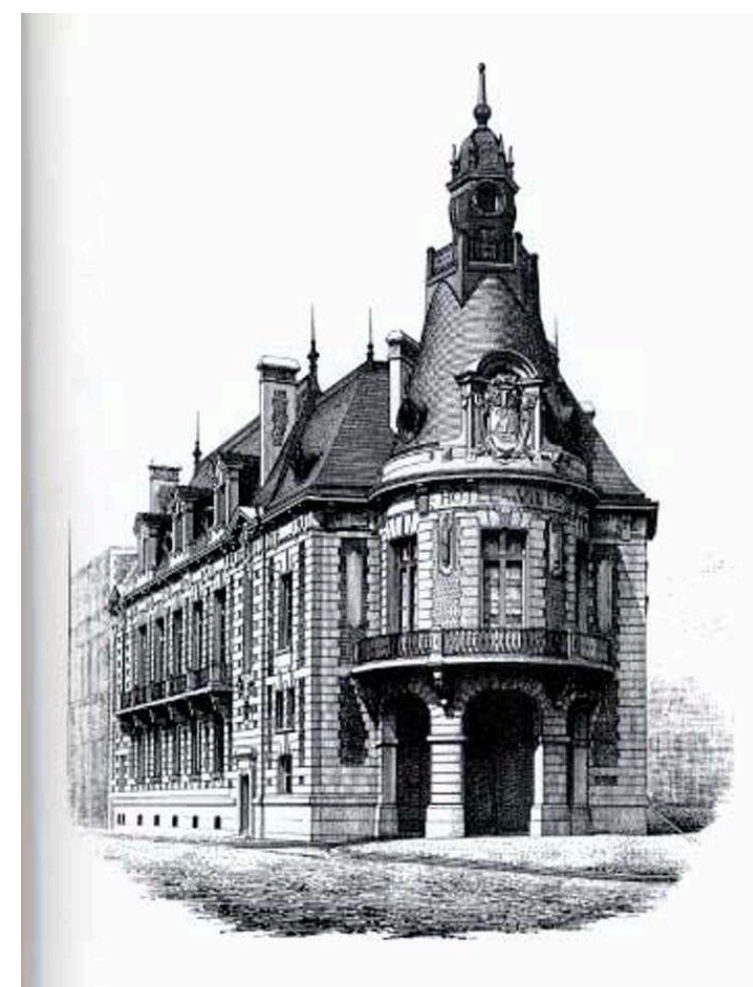

L'hôtel de ville de Trouville de 1911, Laurent et Paul Farge, architectes. Musée de Trouville.

19 Avant de quitter la Belle Epoque, voyons aussi comment certains bâtiments publics s'adaptèrent à la venue de l'automobile. Dessiné par Laurent et Paul Farge en 1911, le nouvel hôtel de ville de Trouville incorpore sur son pan coupé une rotonde ouverte en rez-de-chaussée, formant un porche pour permettre au maire, à ses visiteurs et administrés de descendre de leur automobile à l'abri (fig. $\mathbf{n}^{\circ} \mathbf{1 2}$ ). 
Figure 13

\section{CASIMO DE DEAUVILLE}

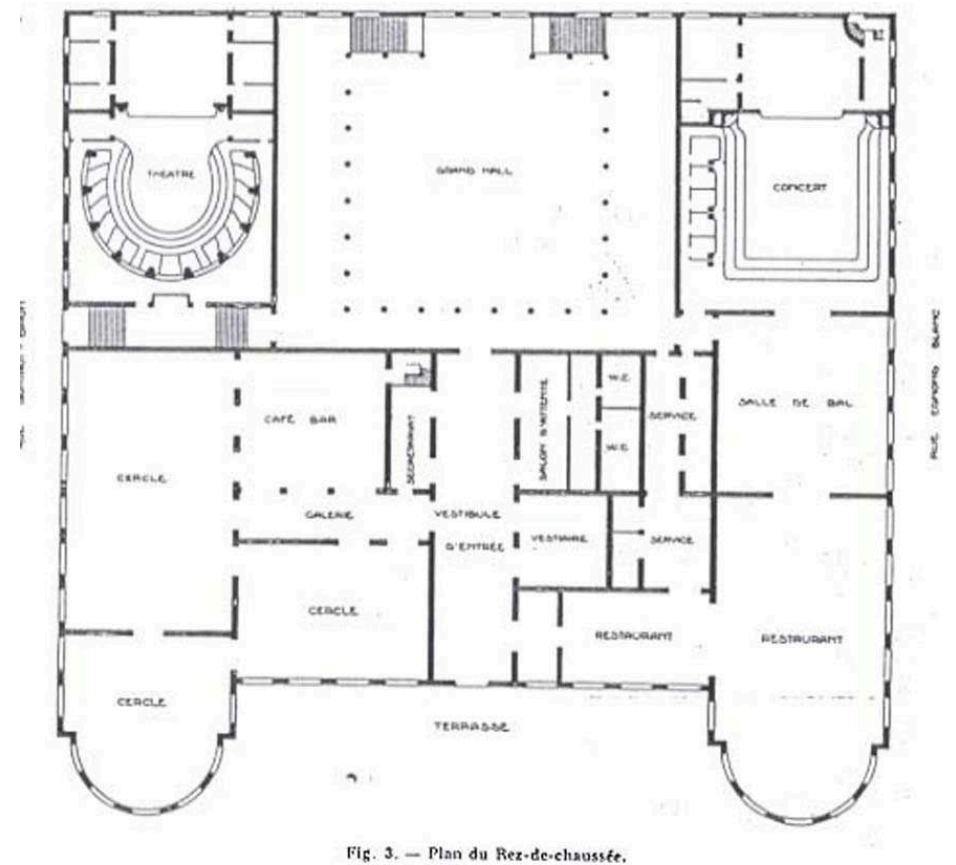

Plan du rez-de-chaussée du casino de Deauville, publié par LE BÉTON ARMÉ en décembre 1912 avant l'adjonction du porche. Collection de I'I.F.A.

Conçu par Georges Wybo et inauguré le 11 juillet 1912, le casino de Deauville, derrière ses apparences Grand Siècle, est un bâtiment d'une grande modernité, construit en béton armé système Hennebique ${ }^{27}$ (fig. $\mathbf{n}^{\circ} \mathbf{1 3}$ ). 
Figure 14

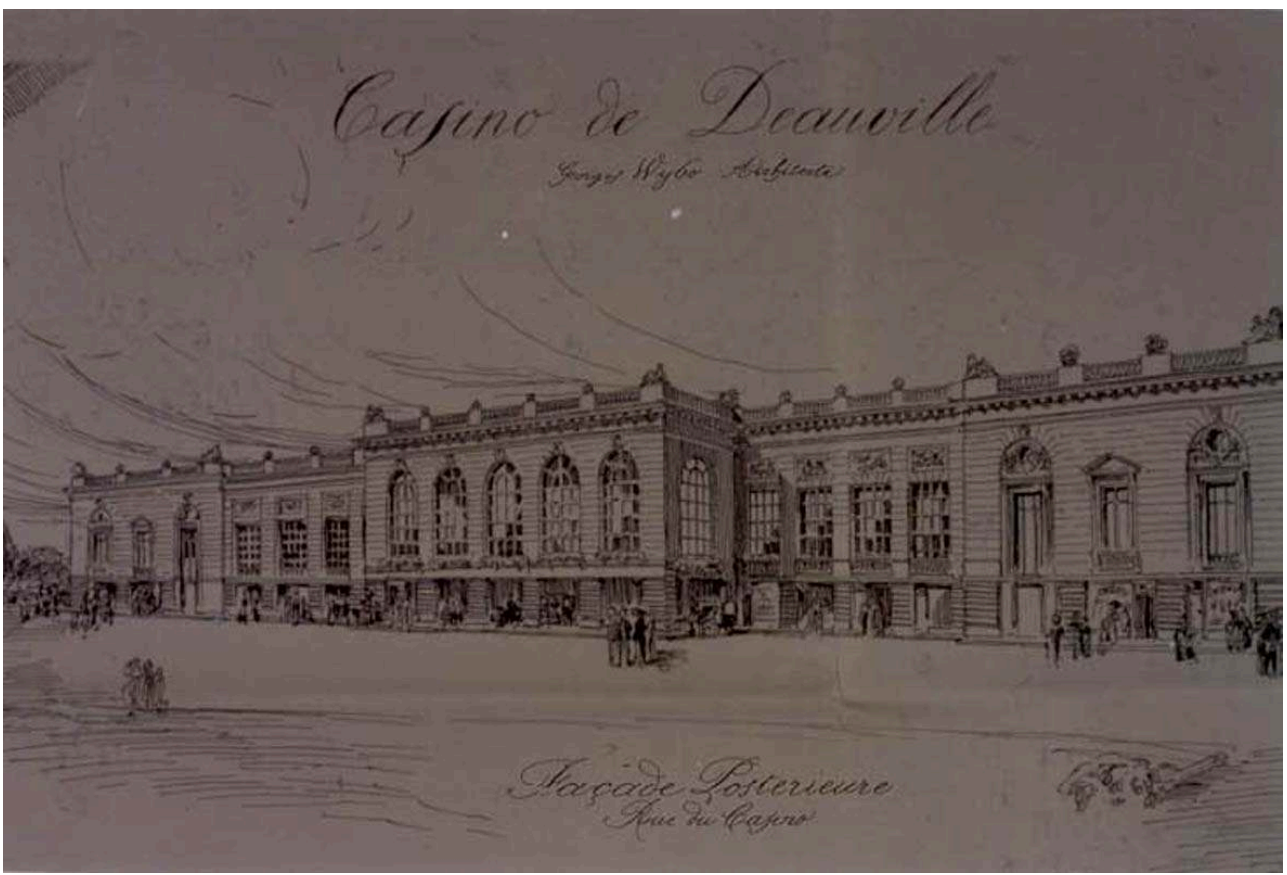

Dessin de Georges Wybo de 1913 : projet du porche sur la façade postérieure du casino. I.F.A., Fonds Georges Wybo (177 IFA).

21 Pour la saison de 1913, il sera agrandi d'un porche d'accès ajouté sur sa façade principale, celle, abritée de la mer, donnant sur la Potinière. Cet avant-corps intègre un passage carrossé, destiné aux automobiles pour débarquer leurs passagers à couvert (fig. $\left.n^{\circ} 14\right)$.

Figure 15

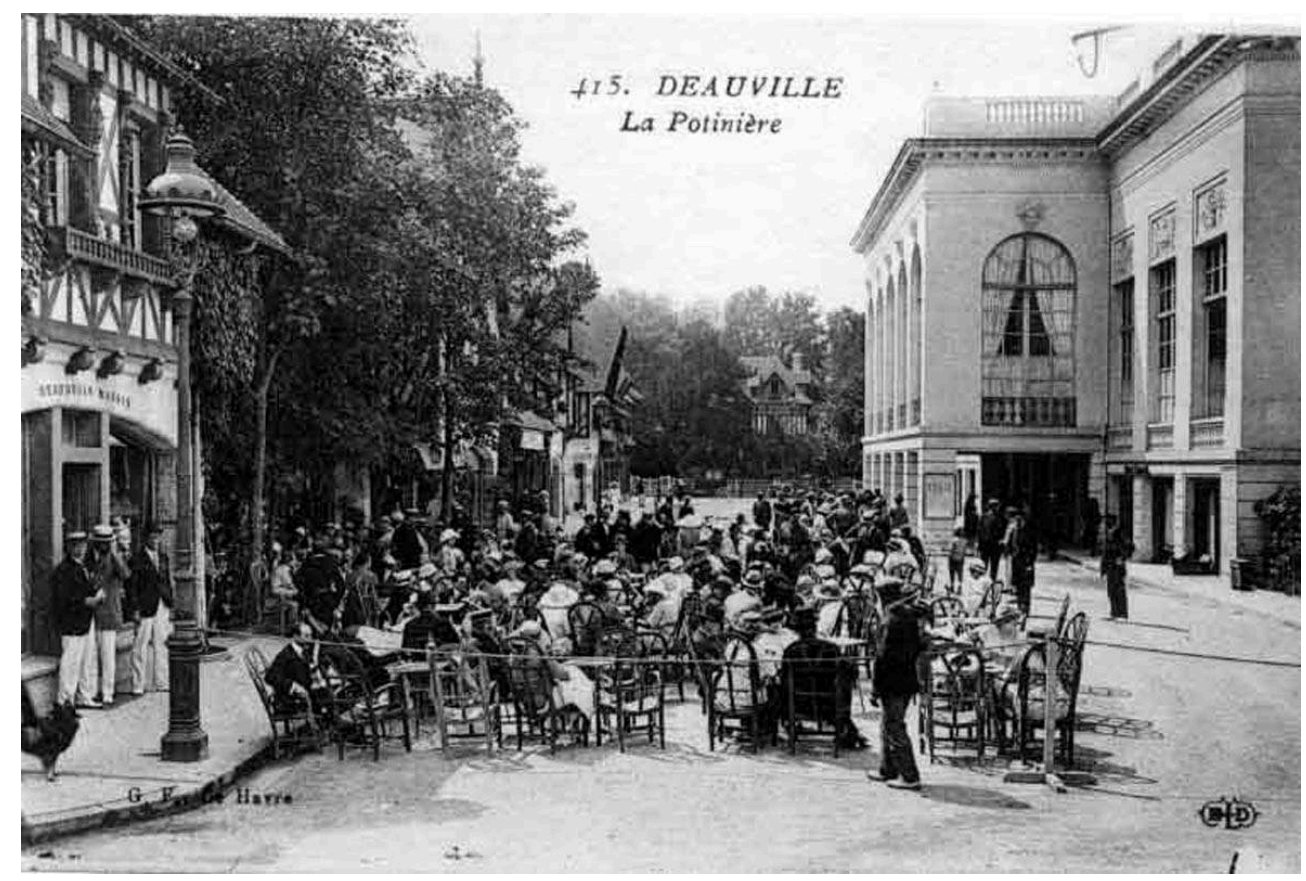

Deauville, La Potinière. Carte postale vers 1913. Coll. part. 
taines grandes églises parisiennes, comme celle de la Trinité, datant du Second Empire, comportent des porches comparables pour abriter des fidèles se rendant à la messe en calèche, mais la mairie de Trouville et le casino de Deauville seraient parmi les tous premiers exemples de ce principe adapté à l'automobile : des bâtiments «drivein » précoces (fig. $\mathbf{n}^{\circ}$ 15).

Flamboyant de nuit de toutes ses 6000 lampes électriques, démultipliées par 1200 miroirs, le casino, meublé par les Grands Magasins du Printemps (2000 chaises et fauteuils), le casino de Deauville est une entreprise de taille considérable, avec 122 employés rien qu'aux jeux ${ }^{28}$. Autour des salles de baccara, de boule et de petits jeux, il incorpore une salle de bal, une salle de music-hall servant également pour des projections cinématographiques, un théâtre, une salle de concert, un grill-room et un bar américain, un restaurant (Les Ambassadeurs), et enfin, de " petits salons avec douches, toilettes et salons de coiffure pour les personnes habitant hors Deauville et désirant s'habiller après les courses pour le dîner; en somme, le confort des grands cercles de Paris $^{29}$ ". L'engouement pour ce luxueux complexe de loisirs est immédiat, et se mesure à la cagnotte et au nombre d'admissions enregistrées : 15000 le 17 août 1913. «Ce n'est plus le Tout-Paris, c'est le Tout-Univers. La file d'automobiles stationnées devant le casino s'allongeait des deux côtés à perte de vue. Les dernières devaient être à Houlgate $^{30} »$.

\section{Citroën}

Interrompant net le développement de l'automobile individuelle, le conflit de 1914-1918 servira d'épreuve tout à fait probante quant aux avantages utilitaires de la locomotion automobile. Dans ses propres productions industrielles, la guerre fera siennes les méthodes tayloristes et fordistes de production de masse qui soutiendront ensuite l'explosion de la production d'automobiles entre les deux guerres. De 94884 en 1919, une voiture pour environ 390 habitants, le parc national passera à 1712900 en 1931, soit une pour 24 habitants. La France est la nation la plus « automobilisée » du Vieux Monde. Sans pouvoir parler encore, avant les années 1950 et l'ère des 2CV et des $4 \mathrm{CV}$, de la « démocratisation » de l'automobile, il s'agit néanmoins, de plus en plus, de voitures « pour tous », construites et commercialisées en grande série.

A Deauville, pour les ventes des yearlings, les courses et les championnats de polo, les chevaux restent encore à l'affiche, mais l'automobile est dorénavant de toutes les festivités, de tous les potins et de toute la production graphique et littéraire de la station:

« Rageusement, on traversa Lisieux sans s'arrêter à ses vieilles maisons de bois, et l'on se dirigea vers Pont L'évêque. Ginette somnolait, ou faisait semblant, et Jacques surveillait l'indicateur de vitesse... ${ }^{31} »$

« Henri Letellier arrive à 135 à l'heure dans une Hispano dont les pneus nouveaux américains semblent gonflés dans les boyaux de M. de Bonnefon... ${ }^{32}$ » «Au bar de chez Ciro's, des gens arrivaient en Hispano ou en Rolls, comme s'ils venaient de très loin... ${ }^{33} »$ 
Figure 16

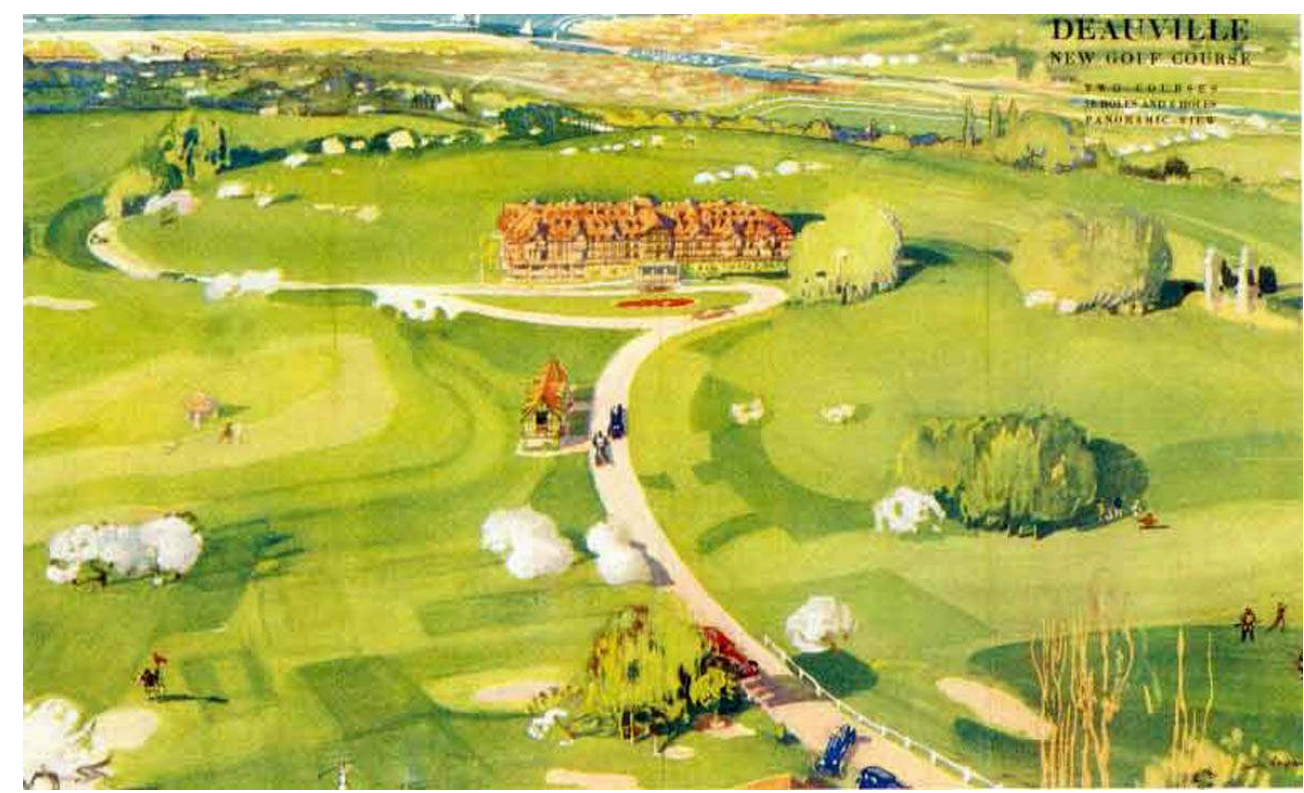

Vue aérienne de l'hôtel du Golf construit par Georges Wybo et E. Lecompte en 1928-1929. Autour de l'hôtel, dont le rez-de-chaussée servait de club-house, les deux parcours étaient dessinés par le spécialiste britannique Simpson. L'aquarelle, en couverture d'une plaquette publicitaire de 1929, est signée d'André Lagrange. Né à Paris en 1889, André Lagrange était le frère de Maurice Lagrange, chef d'agence de Georges Wybo. On lui doit les fresques de 1928 qui décorent les casinos de Trouville et de Deauville (café de la Boule). Musée de Trouville.

L'automobile est prévue aussi dans la construction de tout édifice nouveau, permettant un éloignement progressif de la ville ferro-centrique et l'étalement des stations le long du littoral ou dans l'arrière-pays (fig. $\left.n^{\circ} 16\right)$. 
Figure 17

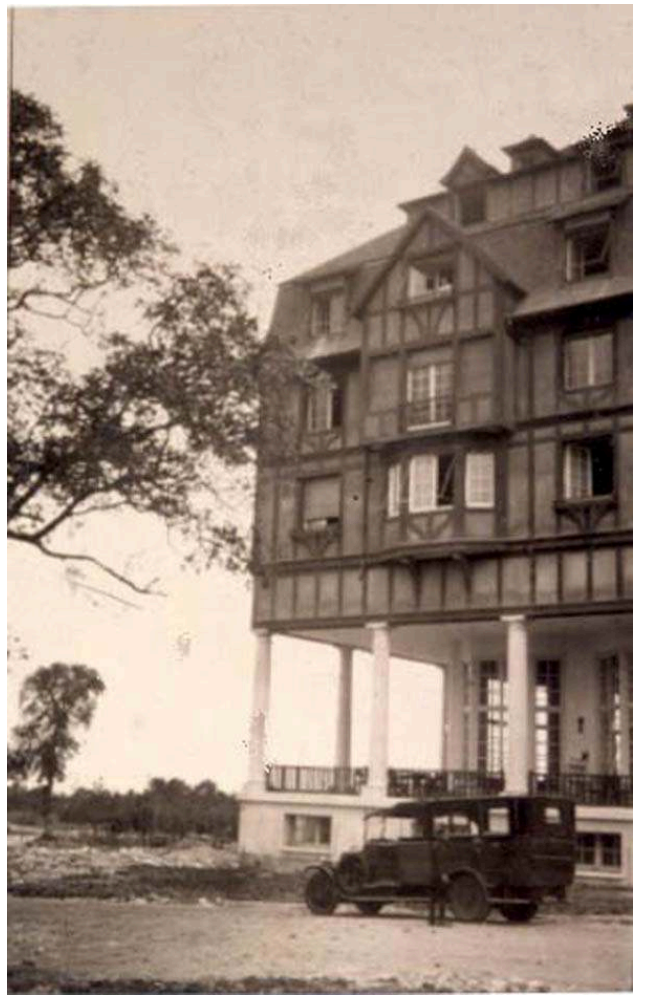

Chantier de construction de I'hôtel du Golf. Photo Georges Wybo, I.F.A., Fonds Georges Wybo (177 IFA).

31 On a remarqué, par exemple, combien la situation même du nouvel hôtel du Golf, soidisant "à trois minutes de la plage ", le fait dépendre exclusivement d'une clientèle motorisée (fig. $\mathbf{n}^{\circ}$ 17). 
Figure 18

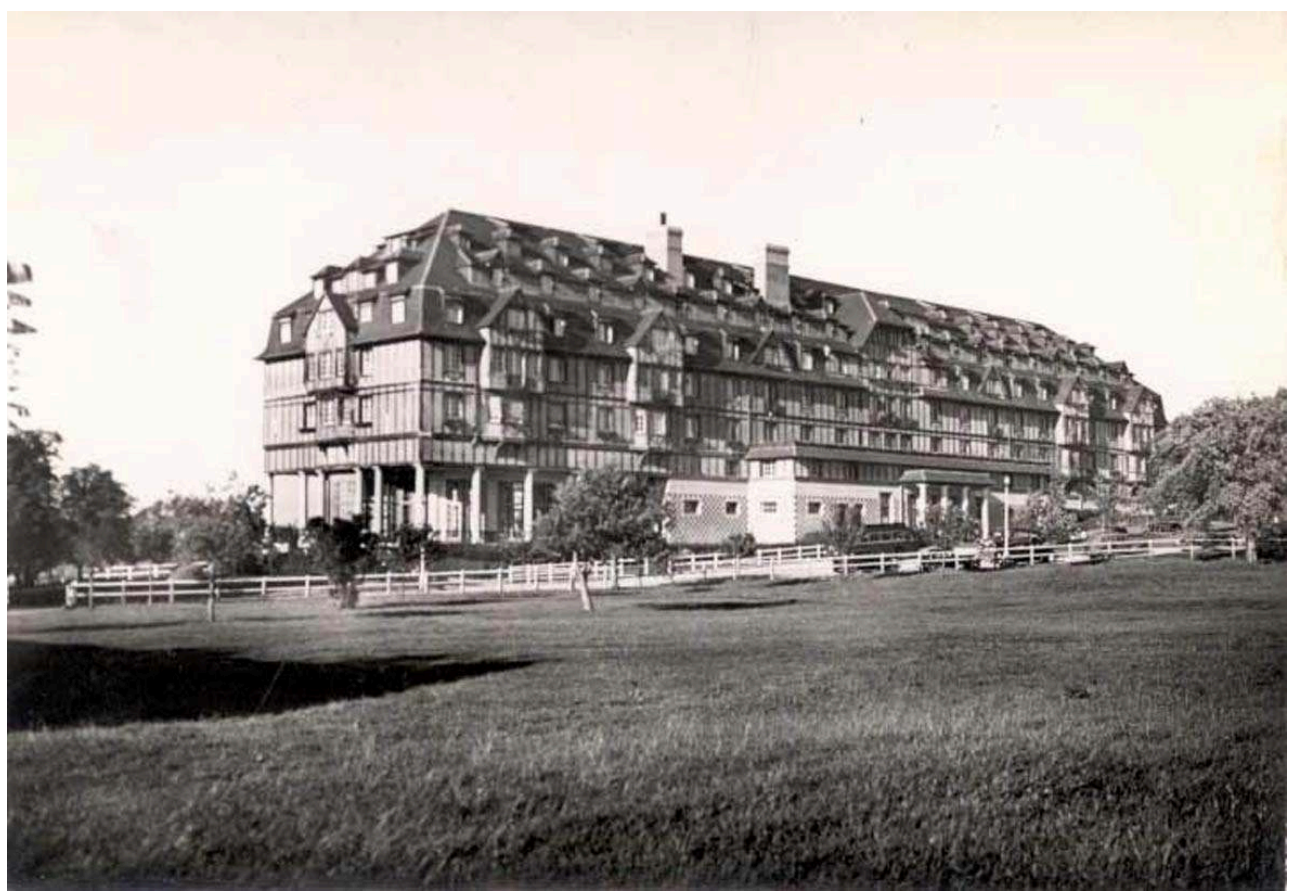

Deauville, la plage fleurie. New Golf Hôtel. Carte postale des années 1950. Coll. part.

Ce palace-hôtel de 250 chambres (fig. $\mathbf{n}^{\circ}$ 18), conçu par Georges Wybo en association avec E. Lecompte, toujours pour la Société des Hôtels et du Casino de Deauville, dirigée depuis la mort de Cornuché par François André, est inauguré en juillet 1929 au milieu des links du Mont Canisy: c'est une nouvelle hybridation monumentale d'éléments stylistiques classiques et normands, dans l'esprit du Royal de 1913, dû à Georges Wybo et Théo Petit. Dans les dépendances néo-normandes de ce "Niou-Golf» se trouvent le " model garage with lodgings for chauffeurs ${ }^{35} »$. 
Figure 19

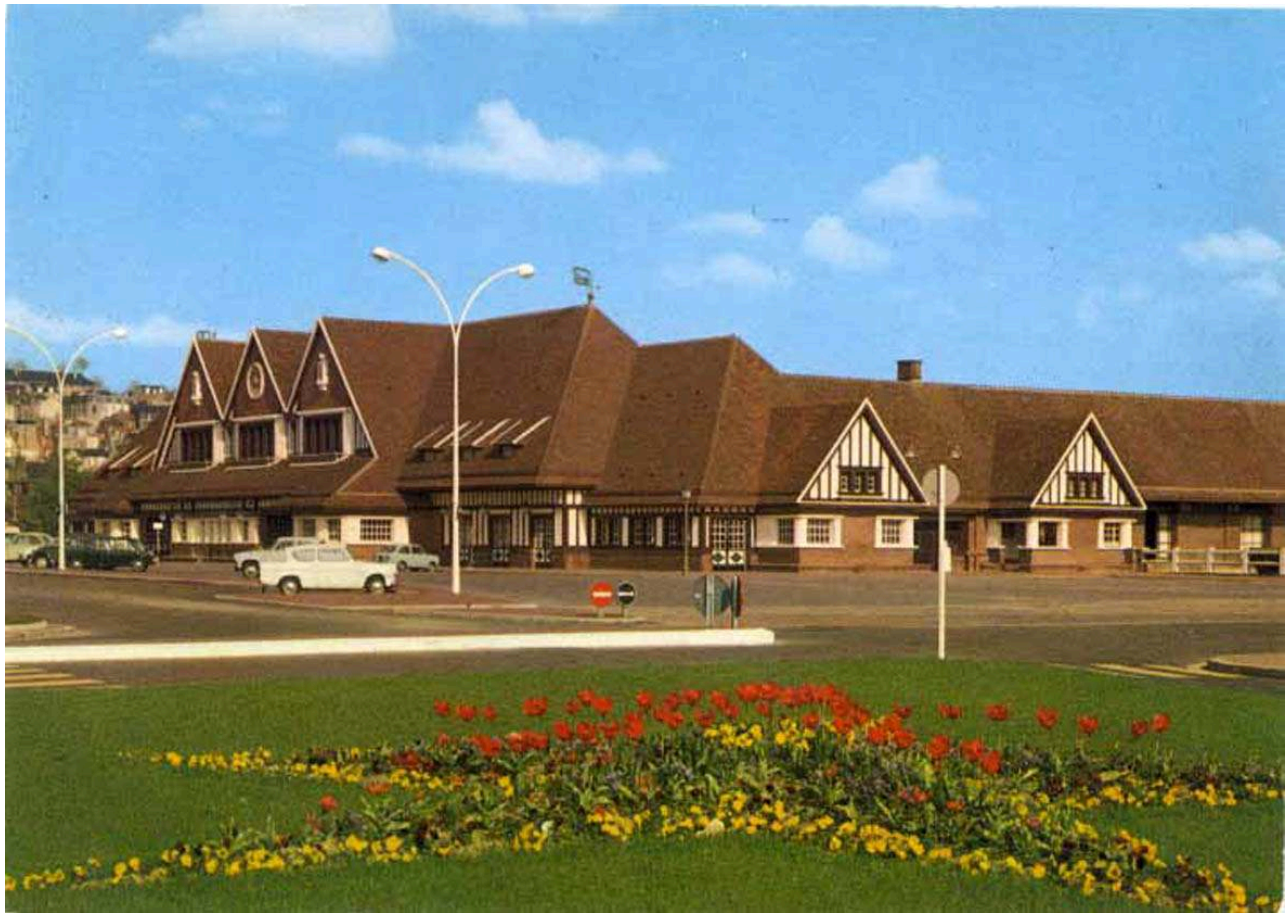

Deauville-Trouville, la gare. Carte postale vers 1965, Coll. part.

Même la nouvelle gare des chemins de fer de Trouville-Deauville, dessinée par Jean Philippot et inaugurée en 1931, doit composer avec l'automobile, non pas tant dans la modernité de son architecture régionaliste, que dans son emplacement. Le nouveau bâtiment est édifié en retrait de l'ancien, créant ainsi ce qui devait être d'abord une sorte de " cour normande » devant la gare, entourée de boutiques et plantée d'arbres, mais cédant rapidement la place à l'évolution et au stationnement de voitures automobiles et de $\operatorname{cars}^{36}$ (fig. $\mathbf{n}^{\circ} \mathbf{1 9}$ ). 
Figure 20

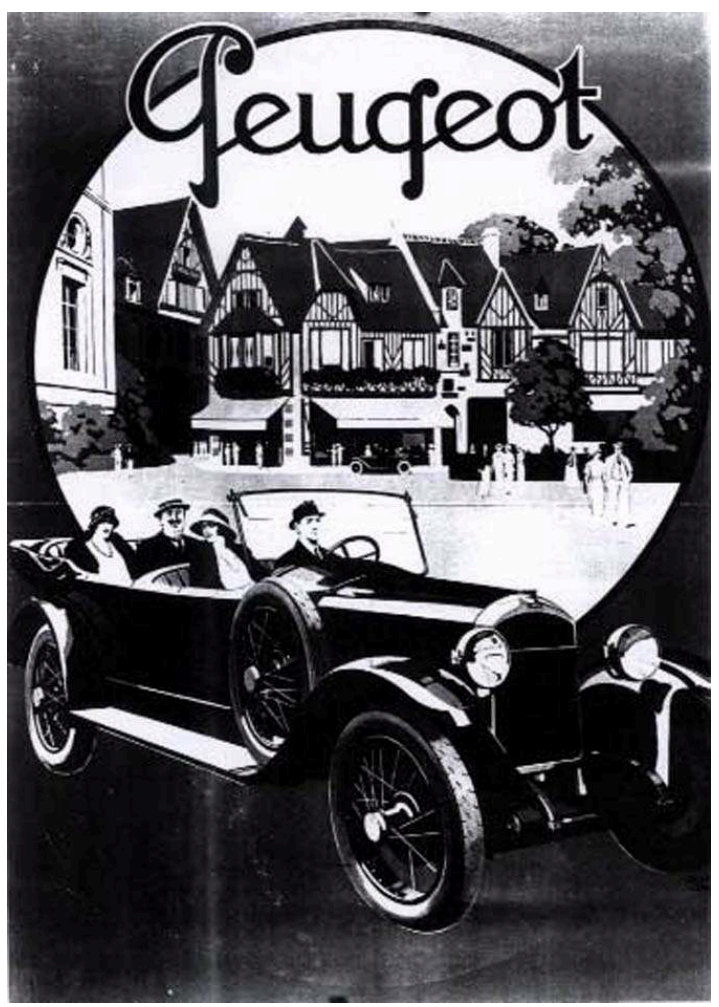

Affiche de Roger Broders pour Peugeot, années 1920. Collection l'Aventure-Peugeot édition La Publicité Française.

Se développe aussi pendant l'entre-deux-guerres un nouveau type de bâtiment commercial, une spécificité du garage en quelque sorte: le magasin d'exposition automobile. Avenue de la République ou rue de l'écluse (Jean-Mermoz) des agences fleurissent pour les marques haut de gamme comme Hispano-Suiza, Voisin et Hotchkiss. Face à la gare, les vieux établissements de Dauquaire et Brunau et de la Société Normande Automobile, devenus concessionnaires respectivement de Renault et de Citroën (le premier subsistant derrière une nouvelle façade de 1934, le deuxième démoli) sont cependant plus représentatifs du commerce automobile d'alors. Celui-ci est très largement dominé par les réseaux intégrés d'agents, concessionnaires et succursales mis en place par les trois grands, Citroën, Renault et Peugeot ${ }^{37}$ (fig. $\left.\mathbf{n}^{\circ} \mathbf{2 0}\right)$. 


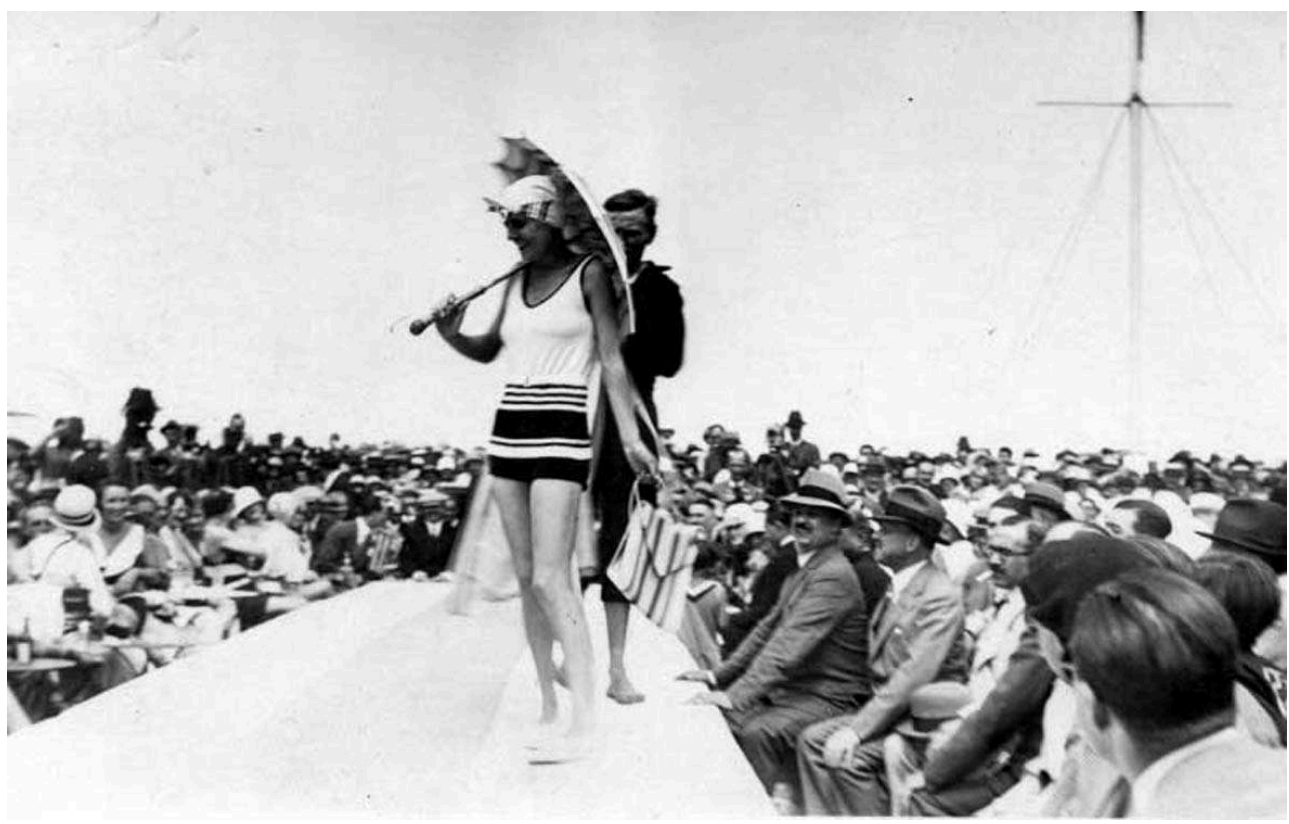

Au Bar du Soleil à Deauville, le 14 juillet 1928 ; à droite, les deux spectateurs en chapeau mou sont Georges Wybo (les mains posées sur l'estrade) et André Citroën. D'après voguE de juillet 1928, l'ensemble qu'ils admirent est signé de Lucien Lelong : costume en jersey avec peignoir doublé éponge, oreiller, sac et couverture assortis, le tout en toile rayée jaune et marron. Carte postale, Coll. part.

Entre Citroën et la ville de Deauville s'est développée une liaison particulière. La présence d'André Citroën est notée dans la station dès 1912, année de son premier pèlerinage aux usines Ford à Detroit. C'est l'époque où le jeune polytechnicien qui s'était lancé dans les engrenages se démenait aussi au sein du conseil d'administration de la Société des Automobiles Mors. C'est aux Ambassadeurs, restaurant du nouveau casino, sur ses terrasses fleuries et en promenade automobile (Mors) qu'il aurait gagné la main de son épouse, Giorgina Bingen, fille d'un banquier de Gênes ${ }^{38}$. Devenu, à la fin des années 1920, le premier constructeur d'automobiles d'Europe, André Citroën passait encore quelques moments de la saison à Deauville, où il louait pour sa famille "Les Abeilles", villa bien en vue, près du Normandy, construite par l'architecte Auguste Bluysen en 1910. Sur les planches ou à l'hippodrome, on croisait Citroën partout, partout on le photographiait. Le samedi 14 juillet 1928, par exemple, il est au Bar du Soleil en compagnie de son architecte Georges Wybo, aux premiers rangs d'un défilé de mode: l'élégance sportive pour Monsieur et Madame à l'heure du bain, présentations de la haute couture parisienne ${ }^{39}$ (fig. $\left.\mathbf{n}^{\circ} \mathbf{2 1}\right)$. 
Figure 22

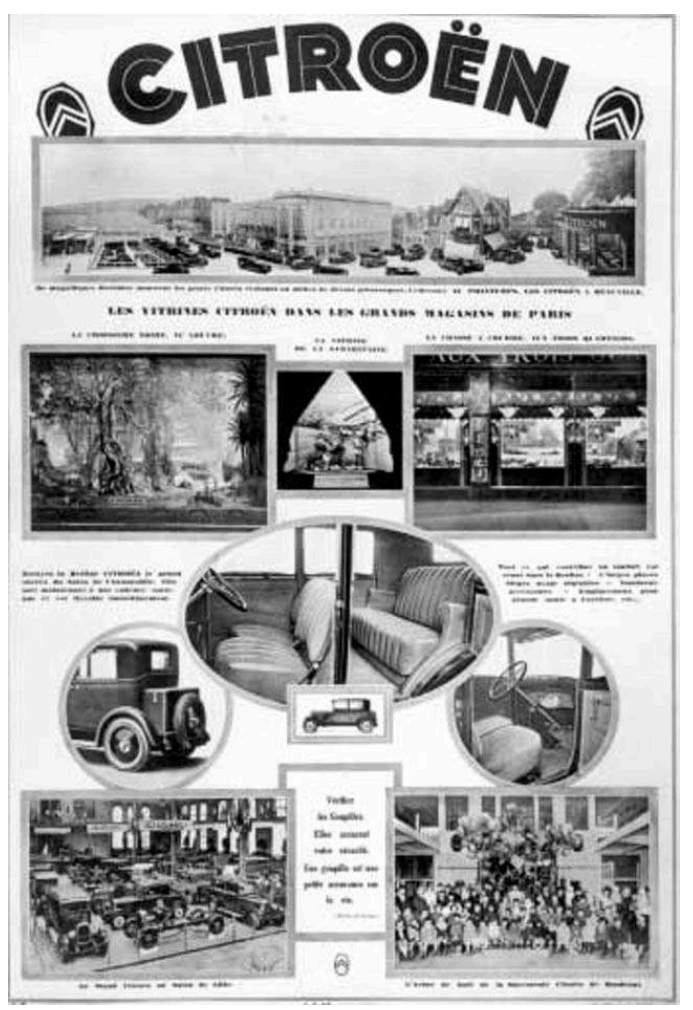

Affiche intérieur Citroën, vers 1927 ; en haut, la vitrine Citroën au Grand Magasin du Printemps, avec le diorama de Deauville.

Collections Citroën @ C Citroën Communication.

On le croquait aussi au « Privé » du casino, où les photographies étaient interdites, et où les millions qu'il aurait gagnés ou perdus la veille alimentaient les potins du lendemain. Entre temps, le long des cabines, s'organisaient de sérieuses courses de "Citroënettes", petits cabriolets $5 \mathrm{CV}$ à pédales, propres à faire rêver de jeunes «citroënnistes». Sur la grève évoluaient des autochenilles Citroën-Kégresse, ces véhicules vedettes de la Croisière noire de 1924-1925, remorquant les baigneurs et les baigneuses vers les flots. A ces images de l'été deauvillais, répercutées à travers tout le réseau commercial Citroën par un bulletin tiré à 4000 exemplaires, d'autres succédaient, en pleine saison des étrennes. La vitrine de Noël de Citroën aux Grands Magasins du Printemps, à l'origine d'une tradition encore animée, présente un diaporama "Les Citroën à Deauville». Sans mer et sans piétons, un défilé de jouets (faux-cabriolets B14) circule entre les maquettes aisément identifiables de quatre édifices de la station, tous œuvres de l'architecte Wybo: l'établissement des bains de mer (celui de 1912, avant les nouveaux bains de Charles Adda), le casino, la succursale du Printemps et le magasin d'exposition Citroën (fig. $\mathbf{n}^{\circ} \mathbf{2 2}$ ). 
Figure 23

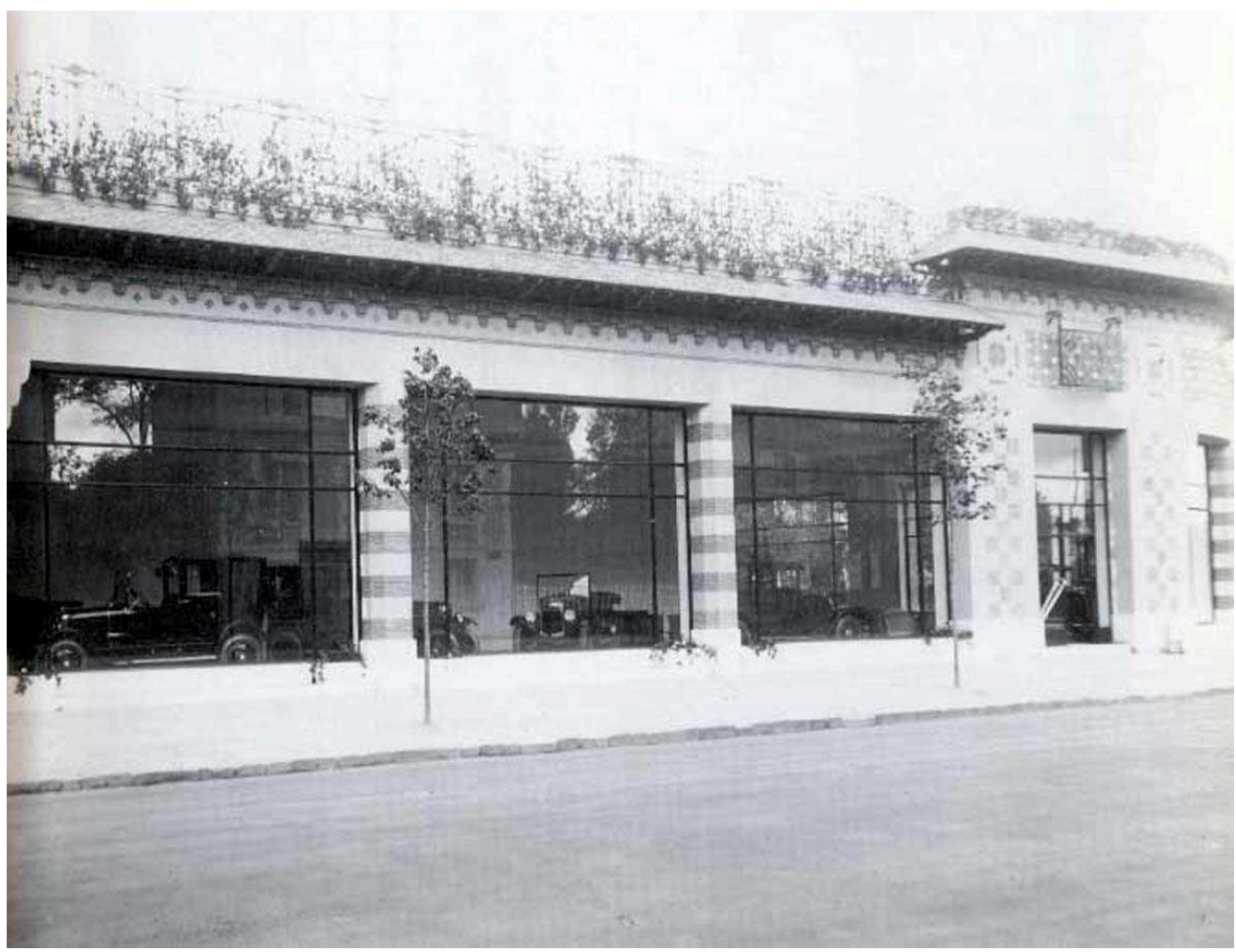

Le magasin d'exposition construit à Deauville en 1926 par Georges Wybo pour la Société Anonyme André Citroën.

Photo Georges Wybo, I.F.A., Fonds Georges Wybo (177 IFA).

Stratégiquement situé derrière le casino, au croisement de l'avenue de la République et de celle de l'Hippodrome (du Général-de-Gaulle), ce magasin de $2000 \mathrm{~m}^{2}$, avec une vingtaine de modèles en exposition, est inauguré le 1er août 1926, devenant aussitôt, nous assure-t-on, «le rendez-vous des élégances internationales de la plage ${ }^{40}$ ». Succursale Citroën, et non pas garage indépendant de concessionnaire, c'était une installation essentiellement publicitaire, brillamment éclairée, encore plus accrocheuse de nuit que de jour, "une fantaisie de grand luxe» écrivait un collaborateur de l'industriel, engloutissant des centaines de millions de francs mais « où l'on ne vendra jamais une seule voiture ${ }^{41} »\left(\right.$ fig. $\left.\mathbf{n}^{\circ} \mathbf{2 3}\right)$. 


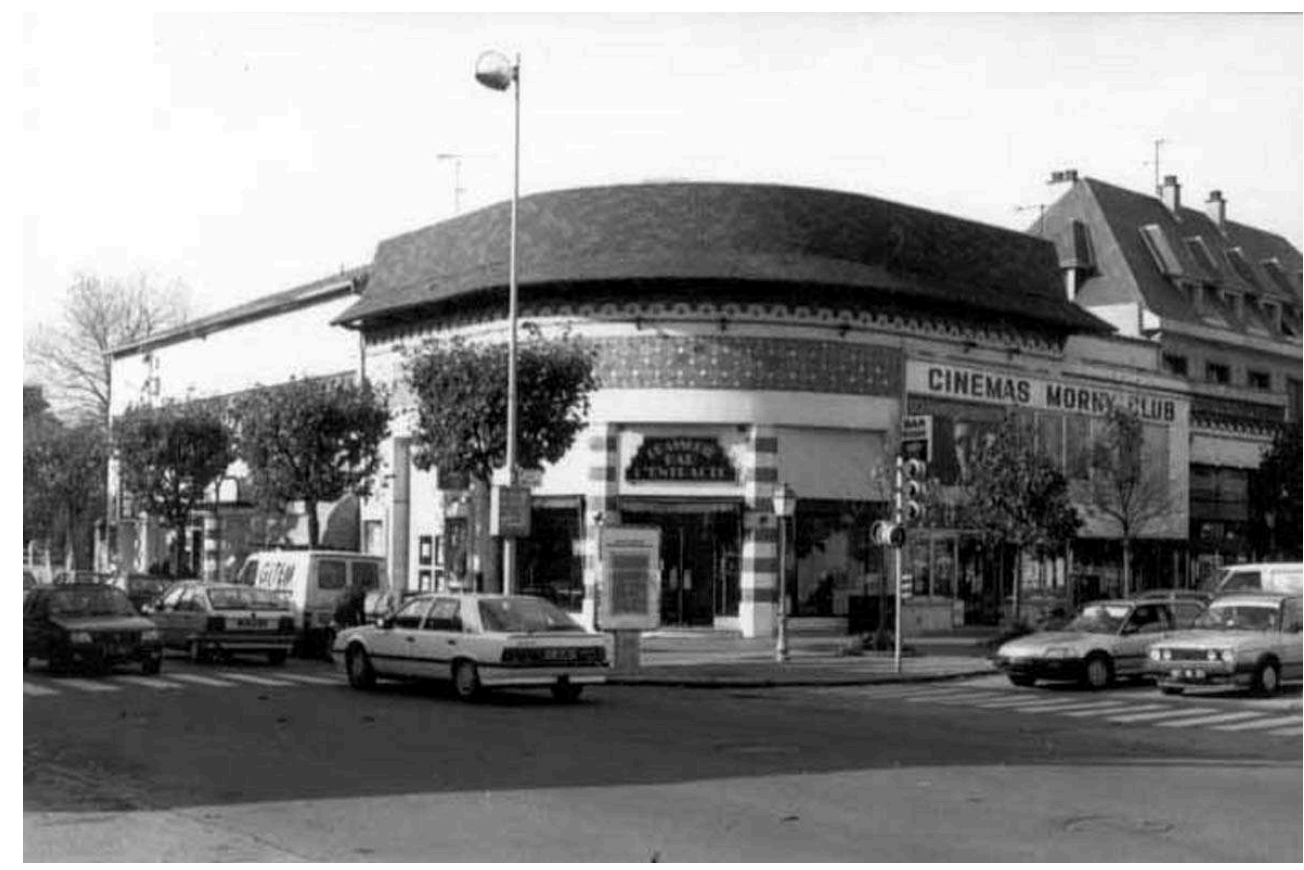

L'ancien magasin Citroën de Deauville, transformé en cinéma en 1946 par l'architecte trouvillais Maurice Vincent.

Phot. P. Smith, 1991 ㄷ P. Smith.

Ainsi, loin d'être un simple moyen de transport (fig. $\mathbf{n}^{\circ}$ 24), et au-delà de son influence directe sur l'urbanisme de la station et sur la forme de ses bâtiments, l'automobile à Deauville est un acteur de premier plan, un élément central et spectaculaire de tous les plaisirs et divertissements de la saison: « Les riches limousines circulent par centaines dans les vastes artères de la Plage Fleurie... C'est la Saison $!^{42} »$.

Aujourd'hui, on le sait, il n'y a plus de saisons, et Deauville vit de congrès, de son Festival de cinéma américain et d'un tourisme de fin de semaine : et si on allait passer le week-end à Deauville ? Grâce à l'autoroute A13, arrivée à la station en 1977 mais commencée à Paris, au tunnel de Saint-Cloud en $1937^{43}$, la côte est à moins de trois heures de la capitale, sa prospérité toujours liée à l'automobile et ses artères toujours encombrées...

\section{NOTES}

1. Toulier, Bernard. Villes d'eaux, stations thermales et balnéaires. Paris : Dexia éditions, Imprimerie nationale éditions, 2002. P. 20.

2. Voir Camard, Florence et Zagrodzki, Christophe. Le Train à l'Affiche, les plus belles affiches ferroviaires françaises. Paris : La Vie du Rail, 1989. Sur 356 affiches reproduites, des années 1880 à 1980, le tiers environ présente des destinations balnéaires. 
3. Sur les origines de l'industrie automobile en France, voir LAUX, James M. In First Gear, The French Automobile industry to 1914. Montréal : McGill-Queen's University Press, 1976. Sur les magasins d'exposition parisiens, voir SMITH, Paul. Les vitrines de l'automobile. Monuments historiques, janvier-février 1991, n 172. P. 57-62.

4. Sur ces salons, voir GOERGER, Michèle. Les espaces d'exposition de l'automobile à Paris. Mémoire inédit. Paris : école d'Architecture de Paris-Belleville, 1987, et BRUNHAMMER, Yvonne et GRANET, Amélie. Les Salons de l'automobile et de l'aviation, décors éphémères d'André Granet. Paris : IFA-Norma, 1993.

5. FONDIN, Jean. Hauts-de-Seine, berceau de l'automobile. Paris: E.T.A.I., Colline de l'Automobile, 1992.

6. Voir CULOT, Maurice et JAKOVLJEVIC, Nada (dirs.). Trouville. Paris : IFA-Mardaga, 1989 (Collection Villes).

7. DESERT, Gabriel. La vie quotidienne sur les plages normandes du Second Empire aux Années Folles. Paris : Hachette. P. 69-71.

8. Sur la carrière de ce personnage, fils d'un marchand de vin du IX ${ }^{\mathrm{e}}$ arrondissement de Paris, né en 1867, fondateur du restaurant Maxim's avant de devenir « le maître incontesté de la cuisine et de l'hôtellerie française ", l'on peut consulter son dossier pour la Légion d'honneur, à laquelle il fut nommé, au grade de chevalier, en janvier 1926, quelques mois avant sa mort: Archives nationales, LH 593/52.

9. SMITH, Paul. Les saisons de l'automobile, in CULOT, Maurice et MIGNOT, Claude (dirs.). Trouville Deauville, société et architectures balnéaires. Paris: IFA-Norma, 1992. P. 68-81. Notre contribution ici reprend en grande partie ce texte d'il y a dix ans, mais essaie d'en renouveler l'iconographie. Sur l'architecture à Deauville, la base Archidoc contient pas moins de 81 références documentaires; avec seulement quatre notices (deux édifices protégés au titre des Monuments historiques et deux sites industriels), la ville est moins bien servie par la base Mérimée, mais le service régional de l'Inventaire y a consacré un itinéraire du patrimoine ( $\left.n^{\circ} 212\right)$ : TOURNOUX, Marie-Noël. Deauville, les styles normands. Caen, Développement culturel en Basse-Normandie, Inventaire général, 1999. Une publication dans la collection «Images du Patrimoine » est également en préparation.

10. DESERT, Gabriel. La vie quotidienne sur les plages normandes du Second Empire aux Années Folles. Paris : Hachette. P. 69.

11. Arrêté cité par Les Echos de Deauville en date du 23 juillet 1914.

12. Chiffres cités par DESERT, Gabriel. La vie quotidienne sur les plages normandes du Second Empire aux Années Folles. Paris : Hachette. P. 70.

13. Le Journal, 11 août 1897.

14. 5000 francs, premier prix pour la course de 1897, 10000 francs pour le grand handicap Omnium de 1899, avec une prime de 4450 francs rajoutée par Michelin et Compagnie pour le premier arrivant muni de ses pneus.

15. Le Journal, 9 septembre 1903.

16. Le Journal, 11 août 1897.

17. Constitué à l'instigation du maire Désiré Le Hoc, président d'honneur, ce syndicat d'initiative alignait un comité d'honneur qui était un véritable " palmarès de personnalités mondaines ». Les statuts du syndicat sont publiés dans le premier numéro de son organe, Les échos de Deauville «La Plage Fleurie ", le $1^{\text {er }}$ mars 1912: «Article $1^{\text {er }}$ : Le syndicat aura surtout pour objectif d'augmenter la durée de la saison et de faire connaître, dans certains milieux sociaux, les sports à la mode qui ont déjà leur organisation à Deauville, tels que courses, polo, concours hippique, golf, tir aux pigeons, tennis, croquet, jeu de paume, etc. ».

18. L'édifice existe toujours, identifiable par la lettre J sur sa façade. Georges Wybo (1880-1943), architecte parisien - son père était bijoutier dans le deuxième arrondissement - a signé ou cosigné une douzaine d'édifices à Deauville. à partir de 1912, il prend la succession de René Binet 
comme architecte en chef des grands magasins du Printemps, pour lesquels il reconstruira le magasin parisien après l'incendie de 1921, et concevra, dans les années 1930, un grand nombre de magasins Prisunic. Associé avec André Citroën à partir de 1916, il est également l'auteur de plusieurs magasins d'exposition pour cette firme ou pour ses concessionnaires, notamment à Bordeaux (1924 et 1928), Caen et Bruxelles (1925), Deauville (1926), Nice (1927), La Baule (garage Minot, 1928), Clermont-Ferrand (1932) et Paris, place de l'Opéra (1932). Grâce à sa fille, Madame Georgette Wybo de Pelluch, ses archives ont été déposées en 1991 au Centre d'archives d'architecture du XX $\mathrm{X}^{\mathrm{e}}$ siècle à l'Institut français d'Architecture (177 IFA). Sur deux aspects de la carrière de Wybo, voir SMITH, Paul. Georges Wybo, architecte à Deauville, dans CULOT, Maurice et MIGNOT, Claude (dirs.). Trouville Deauville, société et architectures balnéaires. Paris : IFANorma, 1992. P. 124-139 et SMITH, Paul. André Citroën, industriel du XX $\mathrm{X}^{\mathrm{e}}$ siècle, dans MARREY, Bernard. Les Bâtisseurs, des moines cisterciens aux capitaines d'industrie. Paris: Le Moniteur, 1997. P. 172-181.

19. Amoureux d'une adolescente de 17 ans, Yola Henriquet, ce Letellier inspira à Colette son roman Gigi, dans lequel il devint Gaston Lachaille, riche héritier des sucres du même nom.

20. DELIENCOURT, Roger et CHENNEBENOIST, Jean. Deauville, son histoire. $2^{\mathrm{e}}$ édition. Honfleur : Imprimerie Marie, 1979 et 1982, 2 vols, (vol. I). P. 212. Darracq investit également sur la Côte d'Azur, notamment dans l'Hôtel Negresco à Nice, fondé en 1912. Claude Laroche l'a également croisé à Hossegor (voir son article dans ce numéro).

21. Pionnier de l'aviation plus-légère-que-l'air, Santos-Dumont fut le premier homme en France à voler dans un avion plus-lourd-que-l'air, sur la pelouse de Bagatelle en 1906. Il n'est guère besoin d'insister ici sur les liens entre l'industrie de l'automobile et celle de l'aviation, mais rappelons que Deauville fut également un haut lieu de l'aviation. La «Grande Quinzaine d'Aviation de la Baie de Seine ", organisée du 25 août au 6 septembre 1910, transforma l'hippodrome de Deauville en « aérodrome ». Santos-Dumont y participait dans sa « Demoiselle », motorisée par ClémentBayard. En août 1913, Deauville devint la «capitale de l'hydraviation » lors d'une course ParisDeauville en hydroaéroplanes et un Concours d'avions marins. Voir NICOLAOU, Stéphane. Deauville 1913 Capitale de l'Hydraviation. Collection «Envols», $n^{\circ} 1$. Paris: éditions de l'Albaron et Musée de l'Air et de l'Espace, 1993.

22. MAZE, Georges. Trouville-Deauville et environs. Rouen : S.A. édition artistique des Guides remboursables de la France pittoresque, s.d. [1913]. P. 59.

23. JOANNE, Paul. Guides-Joanne, France, Normandie. Paris : Hachette, 1904.

24. TURGIS, S. Cent kilomètres autour de Deauville. Deauville : édition du Syndicat d'Initiative de Deauville : Imprimerie E. Desfossés, s.d. [janvier 1913]. P. 5.

25. Délibération du conseil municipal de Deauville, 26 février 1911, Archives municipales, O1D 10. 26. Deauville, Guide local, 1913. Deauville : édition du Syndicat d'initiative, 1913.

27. Voir Le Béton armé, organe des concessionnaires et agents du système Hennebique, décembre 1912, $\mathrm{n}^{\circ}$ 175. P. 179-186.

28. D'après une note relative au mode de partage des pourboires pour la saison de 1914 : Archives départementales du Calvados, dossier sur la police des jeux en M 3696.

29. Deauville, «La Plage Fleurie». Deauville: Syndicat d'Initiative et de Propagande de Deauville, Imprimerie Devambez Gr, 1912. P. 56.

30. Les Echos de Deauville, 18 août 1913.

31. CENILLY, Marcel-Lenoir et HASTINGS, A.T. La Grande semaine ou les loisirs de la Plage Fleurie, itinéraire romanesque. Paris-Deauville : Imprimerie Riou-Reuzé, Rennes, 1920. P. 31. 32. GEORGES-MICHEL, Michel. Nouveau Deauville, Chronique. Paris : Louis Querelle, 1928. P. 146. L'allusion au journaliste Jean de Bonnefon, préfacier d'un livre de dessins de Georges Wybo (Les maisons des champs au pays de France. Paris : Société d'éditions, s.d., [1913]) est devenue assez opaque. 
33. REGNIER, Pierre de. Deauville. (Collection Portrait de la France). Paris : éditions Paul-émile frères, 1927. P. 48.

34. RICHTER, Charles de. La Comédie de Deauville. Paris : éditions « Le Calame », 1929.

35. Deauville Yacht-Club, Hôtel du Golf, The New Deauville. Dépliant promotionnel en langue anglaise de 1929. Paris : Imprimerie G. Gautron (Collections du Musée de Trouville).

36. MIGNOT, Claude. La Gare de Trouville-Deauville, dans CULOT, Maurice et MIGNOT, Claude (dirs.). Trouville Deauville, société et architectures balnéaires. Paris : IFA-Norma, 1992. P. 165-173.

37. Sur le réseau commercial d'André Citroën, voir SMITH, Paul. La Société anonyme André Citroën: architectures d'un réseau commercial, dans GARCON, Anne-Françoise (dir.). L'automobile, son monde et ses réseaux. Rennes : Presses Universitaires de Rennes, 1998. P. 31-45.

38. WOLGENSINGER, Jacques. André Citroën. Paris : Flammarion, 1991. P. 81.

39. Voir SMITH, Paul. Deauville, Au Bar du Soleil, samedi 14 juillet 1928... Athéna sur la Touques, mars 1993, $\mathrm{n}^{\circ} 115$. P. 16-20.

40. Bulletin Citroën commercial, $n^{\circ} 31-32$, juillet-août 1926.

41. ROCHERAND, Charles. L'Histoire d'André Citroën, souvenirs d'une collaboration, 1922-1934. Paris : éditions Lajeunesse, 1938. P. 95. Transformé en cinéma (Le Morny) en 1946, l'édifice subsiste toujours, méconnaissable après sa normandisation par l'architecte Maurice Vincent.

42. Les Echos de Deauville, 18 juillet 1913.

43. PUBLIC-HISTOIRE. Paris-Normandie, une autoroute se souvient... Paris : Presses de l'école nationale des Ponts et Chaussées, 1993.

\section{RÉSUMÉS}

Dès le début du XXe siècle, les stations de la côte normande deviennent une destination privilégiée pour des automobilistes parisiens. L'article prend l'exemple de Deauville, «capitale balnéaire de l'automobile » relancée à la Belle époque par Eugène Cornuché, pour examiner l'impact du nouveau moyen de transport sur l'urbanisme et sur l'architecture de la station. Il s'attache également à cerner le rôle joué à Deauville par les grands constructeurs de l'entredeux-guerres, en particulier André Citroën.

From the beginning of the twentieth century, the seaside resorts of the Normandy coast became a favourite destination for Parisian owners of motor cars. The article looks at the example of Deauville, described as the "seaside capital of the motor car", and examines the impact of the new means of locomotion on the resort's planning and architecture. It also draws attention to the part played by some of France's major manufacturers - André Citroën in particular - in the development and reputation of Deauville between the two wars. 


\section{INDEX}

Mots-clés : A. Debray, Adolphe Clément dit Clément-Bayard (Levallois-Perret), Alberto SantosDumont, Alexandre Darracq, André Citroën, Armand Peugeot, Auguste Bluysen, Automobile Club de France, Biarritz, bois de Boulogne, Bollée, casino, casino de Deauville, Champs-Elysées, côte normande, De Dion-Bouton (Puteaux), Deauville, Decauville, Duc de Morny, E. Deutsch de la Meurthe, E. Lecompte, église de la Trinité, établissement des bains de mer, Eugène Cornuché, Eugène Letellier, Fernand Charron (Courbevoie), Fernard Charron, François André, garage particulier, gare des chemins de fer de Trouville-Deauville, Georges Wybo, Giorgina Bingen, Grand Hôtel Bellevue, Grand Hôtel de la Terrasse, Grand Palais, Grands magasins du Printemps, Hennebique, Henri Letellier, Hispano-Suiza, Hossegor, Hotchkiss, Hôtel de Paris, hôtel de ville, Hôtel des Roches Noires, hôtel du Golf, Jamin, Jean Philippot, La Baule, la Croisière noire, La Potinière de Deauville, Laurent et Paul Farge, Le Touquet, Lebaudy, Louis et Emile Mors, Louis Renault (Billancourt), magasin d'exposition automobile, magasin d'exposition Citroën, Marguerite Duras, Menier, Panhard et Levassor, Renault, Rob Mallet-Stevens, Robert DelaunayBelleville, Rothschild, Société des Hôtels et du Casino de Deauville, succursale du Printemps, Théo Petit, Trouville, villa Les Abeilles, Voisin

\section{AUTEUR}

\section{PAUL SMITH}

Bureau de la recherche et de la méthodologie. Sous-direction des études, de la documentation et de l'Inventaire, Direction de l'architecture et du patrimoine. paul.smith@culture.gouv.fr 